\title{
Second order transport coefficients of nonconformal relativistic fluids in various dimensions from Dp-brane
}

\author{
Chao $\mathrm{Wu}$ \\ MTA Lendület Holographic QFT Group, Wigner Research Centre for Physics, \\ P.O.B. 49, H-1525 Budapest 114, Hungary \\ E-mail: chao.wu@wigner.mta.hu
}

ABSTRACT: We derive all the dynamical second order transport coefficients for Dp-brane with $p$ from 1 to 6 within the framework of fluid/gravity correspondence in this paper. The D5 and D6-brane do not have dual relativistic fluids; D3-brane corresponds to 4dimensional conformal relativistic fluid; D1, D2 and D4-brane separately correspond to nonconformal relativistic fluids of dimensions 2, 3 and 5. The Haack-Yarom relation only exists for Dp-branes with $p$ larger than 2 and is also satisfied by them. We also find that the Romatschke and Kleinert-Probst relations need to be generalized in order to be valid for relativistic fluids of dimensions other than 4 .

KeYwords: Holography and quark-gluon plasmas, AdS-CFT Correspondence, Gaugegravity correspondence, D-branes

ARXIV EPRINT: 1807.08268 


\section{Contents}

1 Introduction $\quad 1$

2 Dimensional reduction from 10 to $p+2$ dimensions $\quad 4$

3 The first order perturbations $\quad 6$

3.1 The D5 and D6-brane case 9

3.2 The Dp-brane case with $1 \leq p \leq 4 \quad 9$

4 The first order stress-energy tensor $\quad 11$

5 The second order perturbations $\quad 12$

$\begin{array}{lll}5.1 \text { General discussions } & 12\end{array}$

$\begin{array}{lll}5.2 & \text { The D3-brane case } & 15\end{array}$

$\begin{array}{ll}5.3 & \text { The D4-brane case } \\ 5.4 & 17\end{array}$

$\begin{array}{ll}5.4 & \text { The D1-brane case } \\ 5.5 & 20\end{array}$

5.5 The D2-brane case 23

6 The second order stress-energy tensor $\quad 26$

$\begin{array}{lll}6.1 & \text { Formulation of the result } & 26\end{array}$

6.2 Relations between second order transport coefficients 30

6.3 Test of Kanitscheider-Skenderis proposal 33

$\begin{array}{lll}6.4 & \text { Dispersion relations } & 35\end{array}$

$\begin{array}{lll}7 & \text { Summary and outlook } & 36\end{array}$

$\begin{array}{ll}\text { A Dimensional reduction of bulk metric } & 37\end{array}$

\section{Introduction}

Relativistic hydrodynamics is a widely used tool in theoretical physics, but its secondderivative behavior is not well understood. This behavior is mostly studied in twoformalisms that are both developed in 2000s. They are the Minkowskian prescription of AdS/CFT duality [1-6] and the fluid/gravity correspondence [7-12]. Much progress has been made in calculating transport coefficients in both of these formalisms, for various kinds of relativistic fluids. We are mainly focusing on the fluids without conserved vector current in this paper. 
To review what is known, we begin by writing down the most general stress-energy tensor for an uncharged, nonconformal, relativistic fluid in $d$ dimensions. This is

$$
\begin{aligned}
T_{\mu \nu} & =\varepsilon u_{\mu} u_{\nu}+\mathfrak{p} P_{\mu \nu}-\left(\eta \cdot 2 \sigma_{\mu \nu}+\zeta P_{\mu \nu} \nabla_{\rho} u^{\rho}\right)+\eta \tau_{\pi} \cdot 2\left(\left\langle D \sigma_{\mu \nu\rangle}+\frac{\sigma_{\mu \nu} \nabla_{\rho} u^{\rho}}{d-1}\right)\right. \\
& +\eta \tau_{\pi}^{*} \cdot \frac{2 \sigma_{\mu \nu} \nabla_{\rho} u^{\rho}}{d-1}+\kappa\left(R_{\langle\mu \nu\rangle}-2 u^{\rho} u^{\sigma} R_{\rho\langle\mu \nu\rangle \sigma}\right)+\kappa^{*} \cdot 2 u^{\rho} u^{\sigma} R_{\rho\langle\mu \nu\rangle \sigma} \\
& +\lambda_{1} \cdot 4 \sigma_{\langle\mu}{ }^{\rho} \sigma_{\nu\rangle \rho}+\lambda_{2} \cdot 2 \sigma_{\langle\mu}{ }^{\rho} \Omega_{\nu\rangle \rho}+\lambda_{3} \Omega_{\langle\mu}{ }^{\rho} \Omega_{\nu\rangle \rho}+\lambda_{4} \nabla_{\langle\mu} \ln s \nabla_{\nu\rangle} \ln s \\
& +P_{\mu \nu}\left(\zeta \tau_{\Pi} D\left(\nabla_{\rho} u^{\rho}\right)+\xi_{1} \cdot 4 \sigma_{\rho \lambda} \sigma^{\rho \lambda}+\xi_{2}\left(\nabla_{\rho} u^{\rho}\right)^{2}+\xi_{3} \Omega_{\rho \lambda} \Omega^{\rho \lambda}\right. \\
& \left.+\xi_{4} P^{\rho \lambda} \nabla_{\rho} \ln s \nabla_{\lambda} \ln s+\xi_{5} R+\xi_{6} u^{\rho} u^{\lambda} R_{\rho \lambda}\right),
\end{aligned}
$$

where $\varepsilon$ is the energy density and $\mathfrak{p}$ is the pressure. The metric $g_{\mu \nu}$ gives the Ricci and Riemann tensor $R_{\mu \nu}$ and $R_{\mu \nu \rho \sigma}$ as usual. $P_{\mu \nu}=g_{\mu \nu}+u_{\mu} u_{\nu}$ is the spatial projection tensor, and $D=u^{\mu} \nabla_{\mu}$ is the temporal derivative, and the spatial-projected traceless symmetric tensor is defined as

$$
A_{\langle\mu \nu\rangle}=P_{\mu}^{\rho} P_{\nu}^{\lambda} A_{(\rho \lambda)}-\frac{1}{d-1} P_{\mu \nu} P^{\rho \lambda} A_{\rho \lambda} .
$$

Matching with our convention in [13], the shear viscous tensor is defined as $\sigma_{\mu \nu}=$ $P_{\mu}^{\rho} P_{\nu}^{\sigma} \nabla_{(\rho} u_{\sigma)}-\frac{1}{d-1} P_{\mu \nu} \nabla_{\rho} u^{\rho}$ where $\nabla_{(\rho} u_{\sigma)}=\frac{1}{2}\left(\nabla_{\rho} u_{\sigma}+\nabla_{\sigma} u_{\rho}\right)$, and the vorticity is defined $\Omega_{\mu \nu}=P_{\mu}^{\rho} P_{\nu}^{\lambda} \nabla_{[\rho} u_{\lambda]}$ with $\nabla_{[\rho} u_{\lambda]}=\frac{1}{2}\left(\nabla_{\rho} u_{\lambda}-\nabla_{\lambda} u_{\rho}\right)$.

Eq. (1.1) has 2 first order transport coefficients (the shear and bulk viscosities $\eta$ and $\zeta$ ) and 15 second order transport coefficients, of which 9 are coefficients of viscous tensors $\left(\eta, \eta \tau_{\pi}, \eta \tau_{\pi}^{*}, \kappa, \kappa^{*}, \lambda_{1,2,3,4}\right)$ and 8 are coefficients of viscous scalars $\left(\zeta, \zeta \tau_{\Pi}\right.$, $\left.\xi_{1,2,3,4,5,6}\right)$. A conformal relativistic fluid has only 6 transport coefficients within second order: $\eta, \eta \tau_{\pi}, \kappa, \lambda_{1,2,3}$. It was shown in [13] that a relativistic fluid living in curved background can have non-zero $\lambda_{4}, \xi_{4,5,6}$ and $\kappa, \kappa^{*}$. The other 9 second order transport coefficients i.e., $\eta \tau_{\pi}, \eta \tau_{\pi}^{*}, \lambda_{1,2,3}, \zeta \tau_{\Pi}, \xi_{1,2,3}$, appear in flat spacetime. The original fluid/gravity correspondence [7] can extract only these 9 transport coefficients.

The study of transport properties within the holographic framework begins with the research on $4 \mathrm{~d}$ conformal relativistic fluid dual to the $\mathrm{AdS}_{5}$ black hole that comes from trivial dimensional reduction on a near-extremal black D3-brane. The real-time prescription of AdS/CFT correspondence was proposed in [1], with which Policastro et al. were able to get the shear viscosity [2] and the sound wave dispersion relation [3] for the $4 \mathrm{~d}$ conformal fluid. The second order transport coefficients were derived several years later in $[4-7,14]$. Then the 1st and 2nd order transport coefficients of conformal relativistic fluid in other dimensions $[10,11,15-18]$, and the coupling constant correction to the transport coefficients caused by higher order terms in bulk action [19-30] were also studied.

The works on nonconformal relativistic hydrodynamics can be divided into two categories, according to the gravity background of the models. The first class is using scalar field deformed $\mathrm{AdS}_{5}$ black holes or the Chamblin-Reall background [31-36] and their results are usually numerical [31-35] or approximate [36]. Thereinto, [31-35] use the AdS/CFT calculation to study the scalar deformed $\mathrm{AdS}_{5}$ black hole and these studies are all numerical, while [36] adopts the method proposed in [37] that the transport coefficients of 
nonconformal fluid can be deduced from a higher dimensional conformal one and use the result from [11] together with the relations among the 2nd order coefficients from [38] to derive the 2nd order transport coefficients for a $5 \mathrm{~d}$ Chamblin-Reall background to first order of $\delta=1-3 c_{s}^{2}$ corrections. The exact results are given in [35].

The second class of works uses instead brane or supergravity backgrounds from string/M-theory and finds analytic results. Because such backgrounds are 10- or 11dimensional, they need to be reduced to lower-dimensional manifold in order to use either AdS/CFT calculation or fluid/gravity correspondence to calculate transport coefficients. References of this kind are $[13,16,37,39-51]$, in which [39-42, 47] are about the relativistic fluid that dual to supergravity backgrounds. And [13, 16, 37, 44-51] are about NS5-brane [44], Dp-brane [16, 37, 46-50] and compactified D4-brane [13, 45, 51]. To be more specific, [37, 44-46, 48, 50, 51] study the first order transport coefficients while $[13,16,47,49]$ investigate the second order ones, in which $[16,47,49]$ calculate the shear and bulk relaxation time $\tau_{\pi}$ and $\tau_{\Pi}$ for Dp-brane and [13] derives all the 7 dynamical second order transport coefficients ${ }^{1}$ for compactified D4-brane.

Of the studies reviewed above, only $[13,16,34-36,47,49]$ are about the 2 nd order transport coefficients of nonconformal relativistic fluids. Our knowledge about this subject is still very limited, especially for Dp-brane backgrounds. In this paper, we would like to derive all the 7 dynamical second order transport coefficients for Dp-brane with $p=1,2, \cdots, 6$, dual to relativistic fluids of dimension $d=p+1=2,3, \cdots, 7$, via fluid/gravity correspondence.

It is said that $\lambda_{3}$ and $\xi_{3}$ can be derived from fluid/gravity duality [13]. Since these two coefficients relate with vortical mode of fluid flow, they will only appear in backgrounds which are not isotropic in all spatial directions. This condition can be satisfied in backgrounds with angular momentum [9] or vector charge [12], for the vector perturbations from metric tensor (backgrounds with angular momentum) are involved with those from vector current (backgrounds with vector charge). So $\lambda_{3}$ and $\xi_{3}$ can not be extracted from branes with $\mathrm{SO}(1, p)$ symmetry in their world-volume directions ( $p$ is the spatial dimension of the brane). Thus both of them are 0 for Dp-brane.

The last part of the Introduction is an overview of the paper.

In section 2, we show the procedure of reducing the 10d near-extremal Dp-brane background to a $(p+2)$-dimensional one. Then we will solve the first order perturbations in section 3, we find that the tensor perturbations of D5- and D6-brane are non-normalizable in subsection 3.1 thus don't have dual fluid. So we only need to solve the Dp-brane with $1 \leq p \leq 4$ of which the first order perturbations are done in subsection 3.2. Then we calculate the first order stress-energy tensor in section 4 and find the results agree with previous studies.

The second order perturbations are solved in section 5. First we derive the second order expanded metric, the constraint equations of the 2nd order viscous terms and the Navier-Stokes equation in subsection 5.1. Then we redo the calculation for the D3-brane

\footnotetext{
${ }^{1}$ The classification of the second order transport coefficients into dynamical and thermodynamical was proposed in [52], according to whether a viscous term contains either $\sigma_{\mu \nu}$ or $\nabla \cdot u$ which can cause entropy increasing.
} 
with our convention in subsection 5.2. The second order perturbations for D4-, D1- and D2-brane are solved in subsection 5.3, 5.4 and 5.5, respectively.

Section 6 will give the final result of the second order stress-energy tensor for Dp-brane. In subsection 6.1 we formulate the final results of the stress-energy tensor and the 2nd order transport coefficients for D4- eq. (6.5), D1- eq. (6.7) and D2-brane eq. (6.9) both in explicit and unified form eq. (6.11). What's more, the gauge theory language of all the transport coefficients are also given in subsection 6.1. In subsection 6.2 we talk about the relations satisfied by the 2 nd order transport coefficients. The most important observation is that the Romatschke and Kleinert-Probst relations need to be generalized when the fluid is not 4-dimensional. The generalized form can be seen in eq. (6.20), eq. (6.25) and eq. (6.23). We give a test on Kanitscheider-Skenderis proposal in subsection 6.3 and calculate the dispersion relations in subsection 6.4.

Section 7 is a summary and some interesting problems for future work will also be mentioned. Appendix A is about the dimensional reduction of bulk metric.

\section{Dimensional reduction from 10 to $p+2$ dimensions}

This section will deal with the reduction from 10d supergravity of Dp-brane to a $(p+2)$ dimensional effective bulk theory. The 10d action of Dp-brane in Einstein frame is

$$
\begin{aligned}
S= & \frac{1}{2 \kappa_{10}^{2}} \int d^{10} x \sqrt{-G}\left[\mathcal{R}-\frac{1}{2}\left(\nabla_{\hat{M}} \phi\right)^{2}-\frac{g_{s}^{2}}{2(8-p) !} e^{\frac{p-3}{2} \phi} F_{8-p}^{2}\right] \\
& -\frac{1}{\kappa_{10}^{2}} \int d^{9} x \sqrt{-H} \mathcal{K}+\frac{1}{\kappa_{10}^{2}} \int d^{9} x \sqrt{-H} \frac{9-p}{2 L_{p}} e^{\frac{3-p}{4(7-p)} \phi} .
\end{aligned}
$$

The first, second and third part of the above action are separately the bulk term, GibbonsHawking surface term and the counter term. The $10 \mathrm{~d}$ surface gravity is $2 \kappa_{10}^{2}=(2 \pi)^{7} g_{s}^{2} l_{s}^{8}$ where $g_{s}$ and $l_{s}$ are separately the string coupling and the string length. In the bulk term, $G_{\hat{M} \hat{N}}$ is the $10 \mathrm{~d}$ metric with $\mathcal{R}$ the corresponding Ricci scalar, $\phi$ is the dilaton with zero VEV and $F_{8-p}$ is the magnetic dual of the Ramond-Ramond field minimally coupled with Dp-brane. The 10d Dp-brane background under the near horizon limit that solves the bulk term of eq. (2.1) is

$$
\begin{aligned}
d s^{2} & =\left(\frac{r}{L_{p}}\right)^{\frac{(7-p)^{2}}{8}}\left(-f(r) d t^{2}+\left(d x^{i}\right)^{2}\right)+\left(\frac{L_{p}}{r}\right)^{\frac{(p+1)(7-p)}{8}}\left(\frac{d r^{2}}{f(r)}+r^{2} d \Omega_{8-p}^{2}\right), \\
e^{\phi} & =\left(\frac{r}{L_{p}}\right)^{\frac{(p-3)(7-p)}{4}} \\
F_{\theta_{1} \cdots \theta_{8-p}} & =g_{s}^{-1} Q_{p} \sqrt{\gamma_{8-p}}
\end{aligned}
$$

where $f(r)=1-\frac{r_{H}^{7-p}}{r^{7-p}}$. The parameter $Q_{p}$ in eq. (2.4) is defined as $Q_{p}=(7-p) L_{p}^{7-p}$ with

$$
L_{p}^{7-p}=\frac{\left(2 \pi l_{s}\right)^{7-p} g_{s} N}{(7-p) \Omega_{8-p}},
$$


where $\Omega_{8-p}=2 \pi^{\frac{9-p}{2}} / \Gamma\left(\frac{9-p}{2}\right)$ is the volume of the $(8-p)$-dimensional unit sphere, and $N$ is the number of the D-branes.

The boundary of eq. (2.2) is at some constant and large value of $r$ with $H_{\hat{M} \hat{N}}=$ $G_{\hat{M} \hat{N}}-\mathbf{n}_{\hat{M}} \mathbf{n}_{\hat{N}}$ the induced metric on it. $\mathbf{n}_{\hat{M}}$ is the unit norm in 10d defined as $\mathbf{n}_{\hat{M}}=$ $\nabla_{\hat{M}} r / \sqrt{G^{\hat{N} \hat{P}} \nabla_{\hat{N}} r \nabla_{\hat{P}} r}$. Then the extrinsic curvature in the Gibbons-Hawking term of eq. (2.1) is $\mathcal{K}=-H^{\hat{M} \hat{N}} \nabla_{\hat{M}} \mathbf{n}_{\hat{N}}$. The counter term for Dp-brane action can refer to e.g., [53], in which $L_{p}$ appears in the denominator just to balance the dimension.

Now we rewrite eq. (2.2) as

$$
d s^{2}=\mathrm{e}^{2 \alpha_{1} A} g_{M N} d x^{M} d x^{N}+L_{p}^{2} \mathrm{e}^{2 \alpha_{2} A} d \Omega_{8-p}^{2}
$$

with its induced boundary metric as

$$
d s^{2}=\mathrm{e}^{2 \alpha_{1} A} h_{M N} d x^{M} d x^{N}+L_{p}^{2} \mathrm{e}^{2 \alpha_{2} A} d \Omega_{8-p}^{2}
$$

to reduce the $10 \mathrm{~d}$ theory described by eqs. $(2.2),(2.1),(2.3),(2.4)$ to a $(p+2)$-dimensional one. The scalar function $A$ can be fixed by comparing eq. (2.6) with eq. (2.2). In order to make the dilaton $\phi$ not coupled with Ricci scalar in the reduced theory, $\alpha_{1}, \alpha_{2}$ should be set as $\alpha_{1}=-\frac{8-p}{p}, \alpha_{2}=1 . g_{M N}$ and $h_{M N}$ are the $(p+2)$-dimensional bulk and induced boundary metric, respectively. The coordinate system is $x^{\hat{M}}=\left\{x^{M}, \theta^{a}\right\}=\left\{x^{\mu}, r, \theta^{a}\right\}$ with $x^{\mu}, r$ and $\theta^{a}$ independent of each other, thus we have $\nabla_{\theta^{a}} r=0$. The $10 \mathrm{~d}$ unit norm reduces to

$$
\mathbf{n}_{\hat{M}}=\frac{\nabla_{\hat{M}} r}{\sqrt{G^{\hat{N} \hat{P}} \nabla_{\hat{N}} r \nabla_{\hat{P}} r}}=\frac{\nabla_{M} r}{\sqrt{G^{N P} \nabla_{N} r \nabla_{P} r}}=\mathrm{e}^{-\frac{8-p}{p} A} n_{M},
$$

where $n_{M}=\frac{\nabla_{M} r}{\sqrt{g^{N P} \nabla_{N} r \nabla_{P} r}}$ is the unit norm in $p+2$ dimensions. Then one also has

$$
\mathcal{K}=-H^{\hat{M} \hat{N}} \nabla_{\hat{M}} \mathbf{n}_{\hat{N}}=\mathrm{e}^{\frac{2(8-p)}{p} A} h^{M N} \nabla_{M}\left(\mathrm{e}^{-\frac{8-p}{p} A} n_{N}\right)=\mathrm{e}^{\frac{(8-p)}{p} A} K
$$

where $K$ is the reduced extrinsic curvature defined as $K=-h^{M N} \nabla_{M} n_{N}$. So the full action for $(p+2)$-dimensional reduced bulk theory is

$$
\begin{aligned}
S= & \frac{1}{2 \kappa_{p+2}^{2}} \int d^{p+2} x \sqrt{-g}\left[R-\frac{1}{2}(\partial \phi)^{2}-\frac{8(8-p)}{p}(\partial A)^{2}+V(\phi, A)\right] \\
& -\frac{1}{\kappa_{p+2}^{2}} \int d^{p+1} x \sqrt{-h} K+\frac{1}{\kappa_{p+2}^{2}} \int d^{p+1} x \sqrt{-h} \frac{9-p}{2 L_{p}}\left(\frac{r}{L_{p}}\right)^{-\frac{(p-3)^{2}}{2 p}}, \\
V(\phi, A)= & \frac{(7-p)(8-p)}{L_{p}^{2}} \mathrm{e}^{-\frac{16}{p} A}-\frac{Q_{p}^{2}}{2 L_{p}^{2(8-p)}} \mathrm{e}^{\frac{p-3}{2} \phi-\frac{2(p+1)(8-p)}{p} A}
\end{aligned}
$$

where $\frac{1}{2 \kappa_{p+2}^{2}}=\frac{L_{p}^{8-p} \Omega_{8-p}}{2 \kappa_{10}^{2}}$ is the surface gravity constant in $(p+2)$-dimensional reduced bulk theory. 
The reduced background which solves eq. (2.10) is

$$
\begin{aligned}
d s^{2} & =\left(\frac{r}{L_{p}}\right)^{\frac{9-p}{p}}\left(-f(r) d t^{2}+\left(d x^{i}\right)^{2}\right)+\left(\frac{r}{L_{p}}\right)^{\frac{p^{2}-8 p+9}{p}} \frac{d r^{2}}{f(r)}, \\
\mathrm{e}^{\phi} & =\left(\frac{r}{L_{p}}\right)^{\frac{(p-3)(7-p)}{4}}, \quad \mathrm{e}^{A}=\left(\frac{r}{L_{p}}\right)^{\frac{(p-3)^{2}}{16}} .
\end{aligned}
$$

Please note that both of the two scalar fields vanish when $p=3$, which is the conformal case that has been solved in [4-7]. The equations of motion (EOM) which can be derived from eq. (2.10) are the Einstein equation and the EOM of scalar fields $\phi$ and $A$ :

$$
\begin{aligned}
E_{M N}-T_{M N} & =0, \\
\nabla^{2} \phi-\frac{(p-3)(7-p)^{2}}{4 L_{p}^{2}} \mathrm{e}^{\frac{p-3}{2} \phi-\frac{2(p+1)(8-p)}{p} A} & =0, \\
\nabla^{2} A-\frac{(7-p)}{L_{p}^{2}} \mathrm{e}^{-\frac{16}{p} A}+\frac{(p+1)(7-p)^{2}}{16 L_{p}^{2}} \mathrm{e}^{\frac{p-3}{2} \phi-\frac{2(p+1)(8-p)}{p} A} & =0 .
\end{aligned}
$$

where $E_{M N}=R_{M N}-\frac{1}{2} g_{M N} R$ is the Einstein tensor and

$$
\begin{aligned}
T_{M N}= & \frac{1}{2}\left(\partial_{M} \phi \partial_{N} \phi-\frac{1}{2} g_{M N}(\partial \phi)^{2}\right)+\frac{8(8-p)}{p}\left(\partial_{M} A \partial_{N} A-\frac{1}{2} g_{M N}(\partial A)^{2}\right) \\
& +\frac{1}{2} g_{M N} V
\end{aligned}
$$

is the energy momentum tensor in $(p+2)$-dimensional spacetime. From the expressions of $\phi$ and $A$ in eq. (2.11) we have $A=\frac{p-3}{4(7-p)} \phi$, which shows that these two scalar fields are not independent thus we only need to solve one of eq. (2.13) and eq. (2.14) to get the scalar perturbations.

\section{The first order perturbations}

We will set $L_{p}=1$ from now on and restore it when we present our results. Now we switch to the Edington-Finkelstein coordinate by $d t=d v-d r /\left(r^{\frac{7-p}{2}} f\right)$, then the metric in eq. (2.11) after coordinate boost becomes

$$
d s^{2}=-r^{\frac{9-p}{p}} f(r) u_{\mu} u_{\nu} d x^{\mu} d x^{\nu}+r^{\frac{9-p}{p}} P_{\mu \nu} d x^{\mu} d x^{\nu}-2 r^{\frac{(p-3)(p-6)}{2 p}} u_{\mu} d x^{\mu} d r .
$$

Following the standard prescription of fluid/gravity correspondence [7], we promote the boost parameters $u^{\mu}$ and $r_{H}$ to be $x^{\mu}$ dependent and expand them to the first order in $\partial_{\mu}$ like in [51], then we get

$$
\begin{aligned}
d s^{2}= & -r^{\frac{9-p}{p}}\left(f-\frac{(7-p) r_{H}^{6-p}}{r^{7-p}} \delta r_{H}\right) d v^{2}+2 r^{\frac{9-p}{p}}(f-1) \delta \beta_{i} d x^{i} d v \\
& +2 r^{\frac{(p-3)(p-6)}{2 p}} d v d r+r^{\frac{9-p}{p}}\left(d x^{i}\right)^{2}-2 r^{\frac{(p-3)(p-6)}{2 p}} \delta \beta_{i} d x^{i} d r .
\end{aligned}
$$


The general form of perturbations are set to be

$$
\begin{aligned}
d s_{\text {gen. pert. }}^{2}= & r^{\frac{9-p}{p}} k(x, r) u_{\mu}(x) u_{\nu}(x) d x^{\mu} d x^{\nu}+2 r^{\frac{9-p}{p}} P_{\mu}^{\rho} w_{\rho}(x, r) u_{\nu} d x^{\mu} d x^{\nu} \\
& +r^{\frac{9-p}{p}}\left(\alpha_{\mu \nu}(x, r)+h(x, r) P_{\mu \nu}(x)\right) d x^{\mu} d x^{\nu}-2 r^{\frac{(p-3)(p-6)}{2 p}} j(x, r) u_{\mu} d x^{\mu} d r .
\end{aligned}
$$

Here we use different conventions for setting the perturbations like $k$ and $w_{\mu}$ from that of $[13,51]$. After expanded to first order, eq. (3.3) becomes

$$
\begin{aligned}
d s_{1 s t \text { pert. }}^{2}= & r^{\frac{9-p}{p}} k^{(1)} d v^{2}-2 r^{\frac{9-p}{p}} w_{i}^{(1)} d x^{i} d v+r^{\frac{9-p}{p}}\left(\alpha_{i j}^{(1)}+h^{(1)} \delta_{i j}\right) d x^{i} d x^{j} \\
& +2 r^{\frac{(p-3)(p-6)}{2 p}} j^{(1)} d v d r
\end{aligned}
$$

in which all the first order perturbations only depend on $r$. This is because the expansion actually happens at a specific point on the boundary (which we set by default the original point). It will apply to all $x^{\mu}$ after we write the solved metric back into covariant form, according to [7]. Eq. (3.4) will be solved in this section.

The perturbations in eq. (3.4) can be grouped into three independent parts according to the transformation rules under $\mathrm{SO}(p)$. The EOM can be divided into the dynamical and constraint equations by the original terminology in [7]. The dynamical equations for Dp-brane are

$$
\begin{aligned}
E_{i j}-\frac{1}{p} \delta_{i j} \delta^{k l} E_{k l}-\left(T_{i j}-\frac{1}{p} \delta_{i j} \delta^{k l} T_{k l}\right) & =0, \\
E_{r i}-T_{r i} & =0, \\
E_{r r}-T_{r r} & =0, \\
\nabla^{2} \phi-\frac{(p-3)(7-p)^{2}}{4} e^{\frac{p-3}{2} \phi-\frac{2(p+1)(8-p)}{p} A} & =0,
\end{aligned}
$$

while the constraint equations are from the vector and scalar sectors:

$$
\begin{aligned}
g^{r 0}\left(E_{0 i}-T_{0 i}\right)+g^{r r}\left(E_{r i}-T_{r i}\right) & =0, \\
g^{r r}\left(E_{r 0}-T_{r 0}\right)+g^{r 0}\left(E_{00}-T_{00}\right) & =0, \\
g^{r r}\left(E_{r r}-T_{r r}\right)+g^{r 0}\left(E_{r 0}-T_{r 0}\right) & =0,
\end{aligned}
$$

of which the nature can be specified from their indices. Whether an equation is dynamical or constrained bases on whether it has second derivative order (with respect to $r$ ) terms of the perturbations: dynamical equations have while the constraint equations do not. Only the EOM of $\phi$ eq. (3.8) is an exception because we do not consider perturbation of $\phi$ in this paper. So eq. (3.8) only contains the first derivative terms of scalar perturbations $h, j$ and $k$.

Now we put eq. (3.2) plus eq. (3.4) into the EOM from eq. (3.5) to eq. (3.11) and get

$$
\begin{aligned}
\partial_{r}\left(r^{8-p} f(r) \partial_{r} \alpha_{i j}(r)\right)+(9-p) r^{\frac{7-p}{2}} \sigma_{i j} & =0, \\
\partial_{r}\left(r^{8-p} \partial_{r} w_{i}^{(1)}\right)-\frac{9-p}{2} r^{\frac{7-p}{2}} \partial_{0} \beta_{i} & =0, \\
2 p r h^{\prime \prime}+p(7-p) h^{\prime}-2(9-p) j^{\prime} & =0, \\
2\left(r^{7-p} k\right)^{\prime}+2 r^{7-p} f j^{\prime}+4(7-p) r^{6-p} j-p r^{7-p} f h^{\prime}-2 r^{\frac{7-p}{2}} \partial \beta & =0,
\end{aligned}
$$


which are the dynamical differential equations and

$$
\begin{aligned}
\frac{1}{r_{H}} \partial_{i} r_{H} & =-\frac{2}{5-p} \partial_{0} \beta_{i}, \\
\frac{1}{r_{H}} \partial_{0} r_{H} & =-\frac{2}{9-p} \partial \beta, \\
\left(r^{7-p} k\right)^{\prime}+2(7-p) r^{6-p} j-\left(p r^{7-p}-\frac{2 p}{9-p} r_{H}^{7-p}\right) h^{\prime}-2 r^{\frac{7-p}{2}} \partial \beta & =0,
\end{aligned}
$$

which are the constraint equations. The differential equation from the EOM of $\phi$ eq. (3.15) does not exist for D3-brane. The vector constraint eq. (3.16) and the first scalar constraint eq. (3.17) are only algebraic equations while the second scalar constraint eq. (3.18) is a differential one which will be used to solve the scalar perturbations. The constraint equations of the algebraic type exist because the viscous terms are not independent of each other. In first order, one has the following viscous terms:

$$
\sigma_{i j}=\partial_{(i} \beta_{j)}-\frac{1}{p} \delta_{i j} \partial \beta, \quad \partial_{0} \beta_{i}, \quad \partial_{i} r_{H}, \quad \partial \beta, \quad \partial_{0} r_{H}
$$

In the above, eq. (3.16) and eq. (3.17) tell us that in vector and scalar part of the viscous tensors, $\partial_{i} r_{H}$ and $\partial_{0} r_{H}$ are not independent of $\partial_{0} \beta_{i}$ and $\partial \beta$, respectively. We will only use the latter two in this paper. Since the perturbations are all $\mathrm{SO}(p)$ invariant, one can set $\alpha_{i j}=$ $F(r) \sigma_{i j}, w_{i}=a(r) \partial_{0} \beta_{i}, h=F_{h}(r) \partial \beta, j=F_{j}(r) \partial \beta$ and $k=F_{k}(r) \partial \beta$. Using eq. (3.14), eq. (3.15) and eq. (3.18), one can eliminate $j, k$ and get the differential equation for $h$

$$
\partial_{r}\left(r^{8-p} f \partial_{r} h\right)+\frac{9-p}{p} r^{\frac{7-p}{2}} \partial \beta=0
$$

Comparing eq. (3.20) with eq. (3.12), one gets

$$
F_{h}=\frac{1}{p} F
$$

which reflects the fact that the scalar perturbation $h$ is the trace part of the perturbation tensor at the first order.

Next we are going to solve the EOM for all the Dp-brane in the first order. We will divide our discussion into the unphysical D5 and D6-brane case and the Dp-brane cases with $1 \leq p \leq 4$. The D3-brane case is actually dual to the renowned $\mathcal{N}=4$ super YangMills plasma, of which the results are already known in $[4,6,7,11]$. The reasons to repeat the calculation for D3-brane are: 1). We will use a different way to solve the perturbations at the first order; 2). At the second order, we will use different conventions to define the viscous terms in order to make them applicable for various dimensions and more convenient to transfer to covariant form. As a result, some of the intermediate results will be different from [7]. 


\subsection{The D5 and D6-brane case}

Solving the tensor perturbation equation (3.12) for $p=5$ and 6 , we get

$$
\begin{array}{ll}
F(r)=C_{2}-\ln \left(r^{2}-r_{H}^{2}\right)+\frac{C_{1}}{2 r_{H}^{2}} \ln \left(1-\frac{r_{H}^{2}}{r^{2}}\right), & \text { (D5-brane) } \\
F(r)=C_{2}-4 r^{\frac{1}{2}}+4 r_{H}^{\frac{1}{2}} \operatorname{artanh} \sqrt{\frac{r}{r_{H}}}+\frac{C_{1}}{r_{H}} \ln \left(1-\frac{r_{H}}{r}\right), & \text { (D6-brane) }
\end{array}
$$

where $C_{1,2}$ are the integration constants. The function "artanh" is sometimes misnamed as "arctanh", the "ar" in artanh means area not arc.

Problems arise in eqs. (3.22), (3.23). One can see that both of these two solutions diverge at $r \rightarrow \infty$ thus do not correspond to physical mode. From $F_{h}=\frac{1}{p} F$ we can conclude that we will not have a physical solution for $h$, either. This means D5 and D6-brane are not dual to any physical relativistic fluid. Similar conclusions have also been made in [47]. The author finds the formula for deriving the shear relaxation time (originally proposed in [14]) vanishes for D5-brane and diverges for D6-brane. The physical reason behind this is also pointed out in [47] that Dp-branes for $p \geq 5$ have instability: the sound speed vanishes for D5-brane and become negative for D6-brane. We will see this instability by calculating the sound speed in section 4 .

The fact that D5 and D6-brane are not dual to any physical relativistic fluid will save us plenty of work, we only need to consider the Dp-brane with $1 \leq p \leq 4$ from now on.

\subsection{The Dp-brane case with $1 \leq p \leq 4$}

We can solve the tensor perturbations for Dp-brane with $2 \leq p \leq 4$ from eq. (3.12) as

$$
\begin{aligned}
F(r)=\frac{2}{5 r_{H}^{3 / 2}}[ & 2 \sin \frac{2 \pi}{5} \arctan \frac{2 \sin \frac{\pi}{5} \sqrt{r r_{H}}}{r-r_{H}}-2 \sin \frac{\pi}{5} \arctan \frac{2 \sin \frac{2 \pi}{5} \sqrt{r r_{H}}}{r-r_{H}} \\
& -2 \cos \frac{2 \pi}{5} \operatorname{artanh} \frac{2 \cos \frac{\pi}{5} \sqrt{r r_{H}}}{r+r_{H}}-2 \cos \frac{\pi}{5} \operatorname{artanh} \frac{2 \cos \frac{2 \pi}{5} \sqrt{r r_{H}}}{r+r_{H}} \\
& \left.+\ln \frac{\left.\left(\sqrt{r}+\sqrt{r_{H}}\right)^{2}\left(r^{4}+r^{3} r_{H}+r^{2} r_{H}^{2}+r r_{H}^{3}+r_{H}^{4}\right)\right], \quad \text { (D2-brane) }}{r^{5}}\right], \quad \text { (D3-brane) } \\
F(r)=\frac{1}{2 r_{H}}\left[2 \arctan \frac{r_{H}}{r}+\ln \frac{\left(r+r_{H}\right)^{2}\left(r^{2}+r_{H}^{2}\right)}{r^{4}}\left[\begin{array}{rl}
r^{6} \\
F(r)=\frac{1}{3 r_{H}^{1 / 2}}
\end{array}\right.\right. & 2 \sqrt{3} \arctan \frac{\sqrt{3 r r_{H}}}{r-r_{H}} \\
& \left.\left.+\ln \frac{\left(\sqrt{r}+\sqrt{r_{H}}\right)^{4}\left(r+\sqrt{r r_{H}}+r_{H}\right)^{2}\left(r^{2}+r r_{H}+r_{H}^{2}\right)}{r^{6}}\right], \quad \text { (D4-bran }\right)
\end{aligned}
$$

Note that D1-brane only has 1 spatial dimension thus can not support tensor perturbation. $F(r)$ for D2-brane is more complicated than the other two cases, yet it can still be cast into a neat form as eq. (3.24). We write the first order tensor perturbation of D3-brane i.e., eq. (3.25) in a different form from that in [7] by using the identity

$$
\frac{\pi}{2}-\arctan x=\arctan \frac{1}{x},
$$

which is easy to verify. 
The D4-brane tensor perturbation eq. (3.26) is the same as in the compactified case [13, 51] which can be shown by first using eq. (3.27) and then the arctangent addition formula. So one can draw the conclusion that compactifying one direction of D4-brane will not effect the coefficients of viscous tensors, i.e., $\eta, \tau_{\pi}$ and $\lambda_{1,2}$ will not change compared with the compactified D4-brane case. This can also be seen from the differential equation of second order tensor perturbation for D4-brane later.

The vector perturbations for Dp-brane with $1 \leq p \leq 4$ can be solved from eq. (3.13) as $w_{i}^{(1)}(r)=a(r) \partial_{0} \beta_{i}$ where we define

$$
a(r)=-\frac{2}{(5-p) r^{\frac{5-p}{2}}}
$$

From the above we can see that the vector perturbation diverges for $p=5$ and can not be normalized for $p=6$. Since we use different convention for setting the vector perturbation in eq. (3.3), that's why the above for $p=4$ is not the same as in [51]. The advantage of this convention is that it can circumvent the appearance of divergent part at the second order, compared with $[7,13,51]$. The vector viscous term $\partial_{0} \beta_{i}$ for D1-brane will reduce to a scalar $\partial_{0} \beta_{1}$ but we will still call it a vector viscous term throughout the paper.

In solving the first order scalar perturbations of Dp-brane, we have some problems on D3-brane case. As it has been pointed out that the EOM of dilaton eq. (3.8) does not exist in D3-brane case and the scalar perturbations need to be solved under the "background field" gauge [7]. Actually, choosing a gauge condition is just adding one more equation factitiously. We also know that the scalar perturbations of D3-brane are trivial, which indicates that they will not be written in a compact form together with the other Dpbranes. In order to express the scalar perturbation solutions of Dp-brane in a unified form, we will use the condition eq. (3.21) for D3-brane. Thus the 3 scalar perturbations of D3-brane can be solved from eq. (3.14), eq. (3.18) together with eq. (3.21).

The other cases can be solved from eq. (3.14), eq. (3.15) and eq. (3.18). Then the 1st order scalar perturbations of Dp-brane with $1 \leq p \leq 4$ can be nicely formulated as

$$
\begin{aligned}
& F_{h}=\frac{1}{p} F, \quad F_{j}=-\frac{2}{9-p} \frac{r^{\frac{9-p}{2}}-r_{H}^{\frac{9-p}{2}}}{r^{7-p}-r_{H}^{7-p}}+\frac{5-p}{2(9-p)} F, \\
& F_{k}=\frac{4}{(9-p) r^{\frac{5-p}{2}}}-\frac{1}{9-p}\left(5-p+\frac{2 r_{H}^{7-p}}{r^{7-p}}\right) F .
\end{aligned}
$$

In D1-brane case $F$ is just $F_{h}$ and we only need to keep in mind that for $p=1 F$ has nothing to do with tensor perturbation. Compared with the solution in compactified D4brane $[13,51], F_{j}$ and $F_{k}$ do not change while $F_{h}$ does. ${ }^{2}$ This is because $h$ relates with the spatial trace part of the perturbation ansatz, as can be seen from eq. (3.4). And the spatial dimension of compactified D4-brane is 3 while it is 4 for D4-brane case here.

\footnotetext{
${ }^{2} F_{k}$ here is $\frac{1}{r^{3}}$ times the $F_{k}$ in $[13,51]$ due to the convention change on the perturbation ansatz eq. (3.3).
} 


\section{The first order stress-energy tensor}

The boundary stress-energy tensor is defined as the large $r$ limit of the following BrownYork tensor

$$
T_{\mu \nu}=\frac{1}{\kappa_{p+2}^{2}} \lim _{r \rightarrow \infty}\left(\frac{r}{L_{p}}\right)^{\frac{(9-p)(p-1)}{2 p}}\left(K_{\mu \nu}-h_{\mu \nu} K-\frac{9-p}{2 L_{p}}\left(\frac{r}{L_{p}}\right)^{-\frac{(p-3)^{2}}{2 p}} h_{\mu \nu}\right),
$$

from which one can calculate the first order stress-energy tensor for Dp-brane:

$$
T_{\mu \nu}=\frac{1}{2 \kappa_{p+2}^{2}}\left[\frac{r_{H}^{7-p}}{L_{p}^{8-p}}\left(\frac{9-p}{2} u_{\mu} u_{\nu}+\frac{5-p}{2} P_{\mu \nu}\right)-\left(\frac{r_{H}}{L_{p}}\right)^{\frac{9-p}{2}}\left(2 \sigma_{\mu \nu}+\frac{2(p-3)^{2}}{p(9-p)} P_{\mu \nu} \partial u\right)\right]
$$

One can extract the energy density, pressure and first order transport coefficients from eq. (4.2) as:

$$
\begin{aligned}
\varepsilon=\frac{1}{2 \kappa_{p+2}^{2}} \frac{9-p}{2} \frac{r_{H}^{7-p}}{L_{p}^{8-p}}, & \mathfrak{p}=\frac{1}{2 \kappa_{p+2}^{2}} \frac{5-p}{2} \frac{r_{H}^{7-p}}{L_{p}^{8-p}}, \\
\eta=\frac{1}{2 \kappa_{p+2}^{2}}\left(\frac{r_{H}}{L_{p}}\right)^{\frac{9-p}{2}}, & \zeta=\frac{1}{2 \kappa_{p+2}^{2}} \frac{2(p-3)^{2}}{p(9-p)}\left(\frac{r_{H}}{L_{p}}\right)^{\frac{9-p}{2}} .
\end{aligned}
$$

From eq. (2.11) one gets the Hawking temperature

$$
T=\frac{(7-p) r_{H}^{\frac{5-p}{2}}}{4 \pi L_{p}^{\frac{7-p}{2}}}
$$

thus the entropy is

$$
s=\frac{\varepsilon+\mathfrak{p}}{T}=\frac{1}{2 \kappa_{p+2}^{2}} 4 \pi\left(\frac{r_{H}}{L_{p}}\right)^{\frac{9-p}{2}} .
$$

Then we have

$$
c_{s}^{2}=\left(\frac{\partial \mathfrak{p}}{\partial \varepsilon}\right)_{s}=\frac{5-p}{9-p}, \quad \frac{\eta}{s}=\frac{1}{4 \pi}, \quad \frac{\zeta}{\eta}=\frac{2(p-3)^{2}}{p(9-p)} .
$$

The above results agree with that of $[37,46,48,50]$. One can see from the sound speed that D5 and D6-brane are indeed unphysical since the sound speed separately become zero and negative for $p=5$ and 6 . 


\section{The second order perturbations}

\subsection{General discussions}

In this section we are going to solve the second order perturbations for Dp-brane with $1 \leq p \leq 4$. The start line is the first order covariant metric valid on the whole boundary:

$$
\begin{aligned}
d s^{2}= & -r^{\frac{9-p}{p}}\left[f\left(r_{H}(x), r\right)-k\left(r_{H}(x), r\right)\right] u_{\mu}(x) u_{\nu}(x) d x^{\mu} d x^{\nu} \\
& +2 r^{\frac{9-p}{p}} P_{\mu}^{\rho}(x, r) w_{\rho}(x, r) u_{\nu}(x) d x^{\mu} d x^{\nu} \\
& +r^{\frac{9-p}{p}}\left[P_{\mu \nu}(x, r)+\alpha_{\mu \nu}\left(r_{H}(x), r\right)+h\left(r_{H}(x), r\right) P_{\mu \nu}(x, r)\right] d x^{\mu} d x^{\nu} \\
& -2 r^{\frac{(p-3)(p-6)}{2 p}}\left[1+j\left(r_{H}(x), r\right)\right] u_{\mu}(x) d x^{\mu} d r .
\end{aligned}
$$

Then expand $r_{H}(x)$ and $\beta_{i}(x)$ to second derivative order like in [13]. If we use $\mathcal{F}$ to denote any of $F, F_{h}, F_{j}$ and $F_{k}$, then $\mathcal{F}$ should be expanded like

$$
\mathcal{F}\left(r_{H}(x), r\right)=\mathcal{F}(r)+\delta \mathcal{F}\left(r_{H}(x), r\right)=\mathcal{F}(r)-\frac{(5-p) \mathcal{F}(r)+2 r \mathcal{F}^{\prime}(r)}{2 r_{H}} \delta r_{H} .
$$

Here $\mathcal{F}(r)$ is $\mathcal{F}\left(r_{H}(x), r\right)$ at the original point $x^{\mu}=0$ and $\delta r_{H}=x^{\mu} \partial_{\mu} r_{H} . \delta \mathcal{F}\left(r_{H}(x), r\right)$ will be denoted shortly as $\delta \mathcal{F}$ in the following. Thus eq. (5.1) should be expanded as

$$
\begin{aligned}
d s^{2}= & -r^{\frac{9-p}{p}}\left[f+(f-1) \delta \beta_{i} \delta \beta_{i}-\frac{(7-p) r_{H}^{6-p}}{r^{7-p}} \delta r_{H}-\frac{(7-p) r_{H}^{6-p}}{2 r^{7-p}} \delta^{2} r_{H}-\frac{(7-p) r_{H}^{6-p}}{r^{7-p}} \delta r_{H}^{(1)}\right. \\
& \left.-\frac{(7-p)(6-p) r_{H}^{5-p}}{2 r^{7-p}}\left(\delta r_{H}\right)^{2}-\left(F_{k}+\delta F_{k}\right) \partial \beta-F_{k}\left(\delta \partial \beta+\delta \beta_{i} \partial_{0} \beta_{i}\right)-2 a(r) \delta \beta_{i} \partial_{0} \beta_{i}\right] d v^{2} \\
& +2 r^{\frac{9-p}{p}}\left[(f-1)\left(\delta \beta_{i}+\frac{1}{2} \delta^{2} \beta_{i}\right)-a\left(\partial_{0} \beta_{i}+\delta \partial_{0} \beta_{i}+\delta \beta_{j} \partial_{j} \beta_{i}\right)-\frac{(7-p) r_{H}^{6-p}}{r^{7-p}} \delta r_{H} \delta \beta_{i}\right. \\
& \left.-F_{k} \partial \beta \delta \beta_{i}-F \delta \beta_{j} \partial_{(i} \beta_{j)}\right] d v d x^{i} \\
+ & 2 r^{\frac{(p-3)(p-6)}{2 p}}\left[1+\left(F_{j}+\delta F_{j}\right) \partial \beta+F_{j}\left(\delta \partial \beta+\delta \beta_{i} \partial_{0} \beta_{i}\right)+\frac{1}{2} \delta \beta_{i} \delta \beta_{i}\right] d v d r \\
+ & r^{\frac{9-p}{p}}\left[\delta_{i j}+(1-f) \delta \beta_{i} \delta \beta_{j}+2 a \delta \beta_{(i} \partial_{|0|} \beta_{j)}+(F+\delta F) \partial_{(i} \beta_{j}\right) \\
& \left.+F\left(\delta \partial_{(i} \beta_{j)}+\delta \beta_{(i} \partial_{|0|} \beta_{j)}\right)\right] d x^{i} d x^{j}-2 r^{\frac{(p-3)(p-6)}{2 p}}\left(\delta \beta_{i}+\frac{1}{2} \delta^{2} \beta_{i}+F_{j} \partial \beta \delta \beta_{i}\right) d x^{i} d r .
\end{aligned}
$$

In the above $\delta \beta_{i}=x^{\mu} \partial_{\mu} \beta_{i}$, similarly as $\delta r_{H}$. We also define $\delta^{2} \beta_{i}=x^{\mu} x^{\nu} \partial_{\mu} \partial_{\nu} \beta_{i}$ and $\delta \beta_{(i} \partial_{|0|} \beta_{j)}=\frac{1}{2}\left(\delta \beta_{i} \partial_{0} \beta_{j}+\delta \beta_{j} \partial_{0} \beta_{i}\right)$. Note after the expansion with respect to the boundary derivative terms, the metric becomes a quadratic function in boundary coordinates $x^{\mu}$ and it is also a complicate function of $r$ via $F, F_{j}$ and $F_{k}$. We will set $r_{H}=1$ hereafter and restore it when we give the results of stress-energy tensors.

Since Einstein equation is second order in derivatives, thus one will get differential equations with many second order derivatives of $r_{H}$ and $\beta_{i}$ which can be written as the spatial 2nd order viscous terms. We list them in table 1 where $\Omega_{i j}=\partial_{[i} \beta_{j]}$ is the spatial component of $\Omega_{\mu \nu}$. Some of the conventions for the 2 nd order spatial viscous terms in this 


\begin{tabular}{|l|l|l|}
\hline Scalars of $\mathrm{SO}(p)$ & Vectors of $\mathrm{SO}(p)$ & \multicolumn{1}{|c|}{ Tensors of $\mathrm{SO}(p)$} \\
\hline $\mathbf{s}_{1}=\frac{1}{r_{H}} \partial_{0}^{2} r_{H}$ & $\mathbf{v}_{1 i}=\frac{1}{r_{H}} \partial_{0} \partial_{i} r_{H}$ & $\mathbf{t}_{1 i j}=\frac{1}{r_{H}} \partial_{i} \partial_{j} r_{H}-\frac{1}{p} \delta_{i j} \mathbf{s}_{3}$ \\
$\mathbf{s}_{2}=\partial_{0} \partial_{i} \beta_{i}$ & $\mathbf{v}_{2 i}=\partial_{0}^{2} \beta_{i}$ & $\mathbf{t}_{2 i j}=\partial_{0} \Omega_{i j}$ \\
$\mathbf{s}_{3}=\frac{1}{r_{H}} \partial_{i}^{2} r_{H}$ & $\mathbf{v}_{4 i}=\partial_{j} \Omega_{i j}$ & $\mathbf{t}_{3 i j}=\partial_{0} \sigma_{i j}$ \\
$\mathfrak{S}_{1}=\partial_{0} \beta_{i} \partial_{0} \beta_{i}$ & $\mathbf{v}_{5 i}=\partial_{j} \sigma_{i j}$ & $\mathfrak{T}_{1 i j}=\partial_{0} \beta_{i} \partial_{0} \beta_{j}-\frac{1}{p} \delta_{i j} \mathfrak{S}_{1}$ \\
$\mathfrak{S}_{3}=\left(\partial_{i} \beta_{i}\right)^{2}$ & $\mathfrak{V}_{1 i}=\partial_{0} \beta_{i} \partial \beta$ & $\mathfrak{T}_{2 i j}=\sigma_{[i}{ }^{k} \Omega_{j] k}$ \\
$\mathfrak{S}_{4}=\Omega_{i j} \Omega_{i j}$ & $\mathfrak{V}_{2 i}=\partial_{0} \beta_{j} \Omega_{i j}$ & $\mathfrak{T}_{3 i j}=\Omega_{i j} \partial \beta$ \\
$\mathfrak{S}_{5}=\sigma_{i j} \sigma_{i j}$ & $\mathfrak{V}_{3 i}=\partial_{0} \beta_{j} \sigma_{i j}$ & $\mathfrak{T}_{4 i j}=\sigma_{i j} \partial \beta$ \\
& & $\mathfrak{T}_{5 i j}=\Omega_{i}{ }^{k} \Omega_{j k}-\frac{1}{p} \delta_{i j} \mathfrak{S}_{4}$ \\
& & $\mathfrak{T}_{6 i j}=\sigma_{i}{ }^{k} \sigma_{j k}-\frac{1}{p} \delta_{i j} \mathfrak{S}_{5}$ \\
& & $\mathfrak{T}_{7 i j}=\sigma_{(i}{ }^{k} \Omega_{j) k}$ \\
\hline
\end{tabular}

Table 1. A list of second derivative order $\mathrm{SO}(p)$ invariant viscous terms for $p \geq 2$. $\mathfrak{T}_{2,5,6}$ are zero when $p=2$.

paper are different from $[7,13]$ because we need to make them appropriate for general value of $p$, not only for the case of $p=3$. Since the viscous terms of D1-brane are very different from the other cases, thus we will list them separately when we talk about D1-brane later.

We would like to stress the changes of table 1 from that in $[7,13]$ : first, we rewrite some terms with $l_{i}=\epsilon_{i j k} \Omega_{i j}$ in terms of $\Omega_{i j}$ since $l_{i}$ only exists for $p=3$ while $\Omega_{i j}$ exists in all cases of $p \geq 2$, e.g. $l_{i} l_{i} \rightarrow \Omega_{i j}^{2}$ and $l_{i} l_{j}-\frac{1}{p} \delta_{i j} l_{k}^{2} \rightarrow \Omega_{i}{ }^{k} \Omega_{j k}-\frac{1}{p} \delta_{i j} \Omega_{k l}^{2}$. Second, we remove $\mathfrak{S}_{2}=l_{i} \partial_{0} \beta_{i}$ and $\mathbf{v}_{3 i}=\partial_{0} l_{i}$ since they both contain $l_{i}$ and can not be rewrite as scalar and vector with the role of $l_{i}$ replaced by $\Omega_{i j}$. In order to be convenient to compare with previous studies, we will not change the ordinal numbers of the other $\operatorname{SO}(p)$ invariant scalars and vectors. Third, we redefine $\mathbf{t}_{2}$ and $\mathfrak{T}_{2,3}$ of $\mathrm{SO}(p)$ tensors in terms of $\Omega_{i j}$ to express the antisymmetric tensor constraint, i.e. eq. (5.14) for general $p$. Thus the viscous tensors in table 1 do not only contain the traceless-symmetric tensors but also the antisymmetric tensors. This is different from that in $[7,13]$. We will suppress the vector and tensor indices of the spatial viscous terms from now on.

The viscous terms listed in table 1 satisfy 6 constraint equations which are the 2nd order counterparts of eqs. (3.16), (3.17). The usage of them is in deriving the Navier-Stokes equation later. These 6 constraints can be solved from $\partial_{\mu} \partial^{\rho} T_{\rho \nu}^{(0)}=0$ by expanding it to second derivative order, the results can be listed as

$$
\begin{array}{r}
\frac{9-p}{2} \partial_{0}^{2} r_{H}+\partial_{0} \partial \beta-\frac{2}{9-p}(\partial \beta)^{2}-\frac{4}{5-p} \partial_{0} \beta_{i} \partial_{0} \beta_{i}=0, \\
\frac{5-p}{2} \partial_{i}^{2} r_{H}+\partial_{0} \partial \beta-\frac{2}{5-p} \partial_{0} \beta_{i} \partial_{0} \beta_{i}-\frac{5-p}{9-p}(\partial \beta)^{2}+\partial_{i} \beta_{j} \partial_{j} \beta_{i}=0, \\
\frac{5-p}{2} \partial_{0} \partial_{i} r_{H}+\partial_{0}^{2} \beta_{i}-\frac{7-p}{9-p} \partial_{0} \beta_{i} \partial \beta+\partial_{0} \beta_{j} \partial_{j} \beta_{i}=0, \\
\frac{9-p}{2} \partial_{0} \partial_{i} r_{H}+\partial_{i} \partial \beta-\frac{2}{5-p} \partial_{0} \beta_{i} \partial \beta-\frac{4}{5-p} \partial_{0} \beta_{j} \partial_{i} \beta_{j}=0,
\end{array}
$$




$$
\begin{aligned}
\partial_{0} \Omega_{i j}-\frac{5-p}{9-p} \Omega_{i j} \partial \beta-\partial_{k} \beta_{[i} \partial_{j]} \beta_{k} & =0 \\
\frac{5-p}{2} \partial_{i} \partial_{j} r_{H}+\partial_{0} \partial_{(i} \beta_{j)}-\frac{2}{5-p} \partial_{0} \beta_{i} \partial_{0} \beta_{j}-\frac{5-p}{9-p} \partial_{(i} \beta_{j)} \partial \beta+\partial_{k} \beta_{(i} \partial_{j)} \beta_{k} & =0
\end{aligned}
$$

Here $T_{\mu \nu}^{(0)}$ is the zeroth derivative order of eq. (4.2), i.e. the thermodynamic part of the 1st order stress-energy tensor of the relativistic fluid. In terms of the viscous $\mathrm{SO}(p)$ invariant terms, the above can be reformulated as

$$
\begin{array}{r}
\mathbf{s}_{1}+\frac{2}{9-p} \mathbf{s}_{2}-\frac{8}{(9-p)(5-p)} \mathfrak{S}_{1}-\frac{4}{(9-p)^{2}} \mathfrak{S}_{3}=0 \\
\mathbf{s}_{2}+\frac{5-p}{2} \mathbf{s}_{3}-\frac{2}{5-p} \mathfrak{S}_{1}+\frac{(p-3)^{2}}{p(9-p)} \mathfrak{S}_{3}-\mathfrak{S}_{4}+\mathfrak{S}_{5}=0 \\
\mathbf{v}_{1}+\frac{2}{5-p} \mathbf{v}_{2}+\frac{2\left(p^{2}-8 p+9\right)}{p(9-p)(5-p)} \mathfrak{V}_{1}-\frac{2}{5-p} \mathfrak{V}_{2}+\frac{2}{5-p} \mathfrak{V}_{3}=0 \\
\mathbf{v}_{1}+\frac{2 p\left(\mathbf{v}_{4}+\mathbf{v}_{5}\right)}{(9-p)(p-1)}-\frac{4(p+2)}{p(9-p)(5-p)} \mathfrak{V}_{1}-\frac{8\left(\mathfrak{V}_{2}+\mathfrak{V}_{3}\right)}{(9-p)(5-p)}=0 \\
\mathbf{t}_{1}+\frac{2}{5-p} \mathbf{t}_{3}-\frac{4}{(5-p)^{2}} \mathfrak{T}_{1}+\frac{2\left(p^{2}-7 p+18\right)}{p(9-p)(5-p)} \mathfrak{T}_{4}-\frac{2}{5-p} \mathfrak{T}_{5}+\frac{2}{5-p} \mathfrak{T}_{6}=0
\end{array}
$$

The above 6 constraints from eq. (5.10) to eq. (5.15) are completely appropriate for D3 and D4-brane. Since all the components of $\mathfrak{T}_{2}, \mathfrak{T}_{5}$ and $\mathfrak{T}_{6}$ are 0 for $p=2$ thus we only need to remove them from eq. (5.14) and eq. (5.15) to get the constraints for D2-brane. The D1-brane needs more changes: first, it does not have tensor part so there should be no tensorial constraints; next, we need to remove the terms $\mathfrak{V}_{2,3}$ from the vector constraint and $\mathfrak{S}_{4,5}$ from scalar constraint because these terms consist of $\sigma_{i j}$ and $\Omega_{i j}$; the last is to use $\mathbf{v}_{4}+\mathbf{v}_{5}=\frac{p-1}{p} \partial_{i} \partial \beta$ to rewrite eq. (5.13) since in this way there is no $p-1$ in the denominator which will blow up as $p=1$. The second order constraints for D1-brane will be given later.

It is interesting to compare the above constraints with that of the compactified D4brane [13] and the D3-brane [7]. Since the convention for the $\mathrm{SO}(p)$ invariant viscous terms are different from that of [7], thus the coefficients of the viscous terms here will also change for D3-brane. Also note that the antisymmetric part of the tensor constraint eq. (5.14) is tensorial here but it is of axial vector type in the cases of D3-brane [7] and compactified D4-brane [13]. Since the spatial dimension for both of these two cases are 3 in which one can define the axial vector $l_{i}$ out of $\Omega_{i j}$, but this can not be done in other dimensions. Thus we write the antisymmetric tensor constraint in terms of three newly defined antisymmetric viscous tensors: ${ }^{3} \mathbf{t}_{2}=\partial_{0} \Omega_{i j}, \mathfrak{T}_{2}=\sigma_{[i}^{k} \Omega_{j] k}$ and $\mathfrak{T}_{3}=\Omega_{i j} \partial \beta$.

There are also two other important equations which can be derived from the full first order stress-energy tensor eq. (4.2), which we denote as $T_{\mu \nu}^{(0+1)}$. They are the Navier-Stokes

\footnotetext{
${ }^{3}$ The old definitions of $\mathbf{t}_{2}$ and $\mathfrak{T}_{2,3}$ contain $l_{i}$ which are not used in calculation in $[7,13,51]$.
} 
equations $\partial^{\mu} T_{\mu \nu}^{(0+1)}=0$ with the index $\nu$ being set to 0 and $i$ :

$$
\begin{aligned}
\frac{1}{r_{H}^{(p-3) / 2}} \partial_{0} r_{H}^{(1)}= & \frac{4(p-3)^{2}}{p(9-p)^{2}(7-p)} \mathfrak{S}_{3}+\frac{4}{(9-p)(7-p)} \mathfrak{S}_{5}, \\
\frac{1}{r_{H}^{(p-3) / 2}} \partial_{i} r_{H}^{(1)}= & \frac{4(p-3)^{2} \mathbf{v}_{4}+16 p \mathbf{v}_{5}}{(9-p)(7-p)(5-p)(p-1)}+\frac{2(p-1)\left(p^{2}-22 p+77\right)}{p(9-p)(7-p)(5-p)^{2}} \mathfrak{V}_{1} \\
& -\frac{2(19-3 p)}{(9-p)(7-p)(5-p)} \mathfrak{V}_{2}-\frac{2\left(p^{2}-14 p+77\right)}{(9-p)(7-p)(5-p)^{2}} \mathfrak{V}_{3} .
\end{aligned}
$$

The above two equations are suitable for $2 \leq p \leq 4$. As what we have specified for the 2 nd order constraints of D1-brane case, we need to remove $\mathfrak{S}_{5}$ and $\mathfrak{V}_{2,3}$ first and then recast the terms with $\mathbf{v}_{4,5}$ into $\partial_{1}^{2} \beta_{1}$. The explicit form of the Navier-Stokes equations for $p=1$ will be offered later. As we have said that in deriving these two equations, we need to use the constraints from eq. (5.4) to eq. (5.9). The Navier-Stokes equations will be used in deriving the differential equations for the 2 nd order perturbations. They can also be got from the bulk vector and scalar constraint equations at second order, which will be specified later.

Following $[7,13]$, the second order perturbations can be solved by integrations as

$$
\begin{aligned}
\alpha_{i j}^{(2)} \text { or } h^{(2)} & =\int_{r}^{\infty} \frac{-1}{x^{8-p} f} d x \int_{1}^{x} S_{\alpha, h}(y) d y, \\
j^{(2)} \text { or } k^{(2)} & =-\int_{r}^{\infty} S_{j, k}(x) d x \\
w_{i}^{(2)} & =\int_{r}^{\infty} \frac{1}{x^{8-p}} d x \int_{x}^{\infty} S_{w}(y) d y
\end{aligned}
$$

where $S_{\#}$ with $\#=\{\alpha, w, h, j, k\}$ are the source terms which can be easily understood as the right hand side of the perturbation equations. The above integrations are suitable for the nonconformal branes, i.e., $p=1,2,4$. For $p=3, h^{(2)}$ needs to be integrated in the same way as $w_{i}^{(2)}$ but not like $\alpha_{i j}^{(2)}$ since the scalar part of D3-brane is trivial. While the other perturbations of D3-brane are still integrated in the same way as the other Dp-branes.

\subsection{The D3-brane case}

Although the second order transport coefficients of D3-brane has been studied in [7], we still would like to redo the calculation here. The reason is that we use different conventions, thus the intermediate steps of the calculation will also change. Further more, the D3-brane calculation can help us to understand the nonconformal cases.

At the second order there is no simple relation between $h$ and $\alpha_{i j}$ like eq. (3.21). Since in D3-brane case we do not have the dilaton equation and one only has eq. (3.7) and eq. (3.11) at hand to solve $h, j$ and $k$, which are not enough. Thus we need a gauge condition to eliminate one of the degrees of freedom. Here we use the "background field" gauge $g^{(0) M N} g_{M N}^{(n)}=0$ from [7], which gives $j^{(n)}=-\frac{3}{2} h^{(n)}$, where $(n)$ specifies the order of each perturbation. With this we can solve $h, j, k$ for D3-brane at first order as

$$
h^{(1)}=j^{(1)}=0, \quad k^{(1)}=\frac{2}{3 r} \partial \beta .
$$


Since $F_{h, j, k}$ are trivial for D3-brane under the background field gauge, the second order expanded metric of D3-brane can not be got by simply setting $p=3$ in eq. (5.3), but needs to be derived separately. The result is:

$$
\begin{aligned}
d s^{2}=-r^{2}\left[f+(f-1) \delta \beta_{i} \delta \beta_{i}-\frac{4 r_{H}^{3}}{r^{4}} \delta r_{H}-\frac{2 r_{H}^{3}}{r^{4}} \delta^{2} r_{H}-\frac{4 r_{H}^{3}}{r^{4}} \delta r_{H}^{(1)}-\frac{6 r_{H}^{2}}{r^{4}}\left(\delta r_{H}\right)^{2}\right. \\
\left.\quad-F_{k} \partial \beta-F_{k}\left(\delta \partial \beta+\delta \beta_{i} \partial_{0} \beta_{i}\right)-2 a \delta \beta_{i} \partial_{0} \beta_{i}\right] d v^{2} \\
+2 r^{2}\left[(f-1)\left(\delta \beta_{i}+\frac{1}{2} \delta^{2} \beta_{i}\right)-a\left(\partial_{0} \beta_{i}+\delta \partial_{0} \beta_{i}+\delta \beta_{j} \partial_{j} \beta_{i}\right)-\frac{4 r_{H}^{3}}{r^{4}} \delta r_{H} \delta \beta_{i}\right. \\
\left.\quad-F_{k} \partial \beta \delta \beta_{i}-F \delta \beta_{j} \partial_{(i} \beta_{j}+\frac{1}{3} F \partial \beta \delta \beta_{i}\right] d v d x^{i}+2\left(1+\frac{1}{2} \delta \beta_{i} \delta \beta_{i}\right) d v d r \\
+r^{2}\left[\left(1-\frac{1}{3}(F+\delta F) \partial \beta-\frac{1}{3} F\left(\delta \partial \beta+\delta \beta_{k} \partial_{0} \beta_{k}\right)\right) \delta_{i j}+(1-f) \delta \beta_{i} \delta \beta_{j}\right. \\
\left.\quad+2 a \delta \beta_{(i} \partial_{|0|} \beta_{j)}+(F+\delta F) \partial_{(i} \beta_{j)}+F\left(\delta \partial_{(i} \beta_{j)}+\delta \beta_{(i} \partial_{|0|} \beta_{j)}\right)\right] d x^{i} d x^{j} \\
-2\left(\delta \beta_{i}+\frac{1}{2} \delta^{2} \beta_{i}\right) d x^{i} d r
\end{aligned}
$$

The metric of the perturbative ansatz at 2nd order has the same form as eq. (3.4) with the first order perturbations changing to second order ones. By substituting eq. (5.22) and the 2nd order perturbations into the EOM eq. (3.5) to eq. (3.7) and the constraint equations (3.9), (3.10), (3.11), we can get ${ }^{4}$

$$
\begin{aligned}
\partial_{r}\left(r^{5} f \partial_{r} \alpha_{i j}^{(2)}\right)= & \left(2 r-3 r^{2} F-2 r^{3} F^{\prime}\right)\left(\mathbf{t}_{3}+\mathfrak{T}_{1}+\frac{\mathfrak{T}_{4}}{3}\right)-\left(\frac{4}{r^{3}}+4 r\right) \mathfrak{T}_{5} \\
& +\left(4 r-6 r^{2} F+r^{5} f F^{\prime 2}\right) \mathfrak{T}_{6}-\left(4 r+6 r^{2} F+4 r^{3} F^{\prime}\right) \mathfrak{T}_{7}, \\
\partial_{r}\left(r^{5} \partial_{r} w_{i}^{(2)}\right)= & -2 r \mathbf{v}_{4}+r^{3} F^{\prime} \mathbf{v}_{5}+\left(\frac{2}{3} r+\frac{5}{9} r^{3} F^{\prime}\right) \mathfrak{V}_{1}+\left(r-\frac{5}{6} r^{3} F^{\prime}\right) \mathfrak{V}_{2} \\
& -\left(r+\frac{11}{6} r^{3} F^{\prime}\right) \mathfrak{V}_{3}, \\
r h_{(2)}^{\prime \prime}+2 h_{(2)}^{\prime}-2 j_{(2)}^{\prime}= & \frac{2}{3 r^{3}} \mathfrak{S}_{4}+\left(\frac{1}{6} r F^{\prime 2}+\frac{2}{3} F F^{\prime}+\frac{1}{3} r F F^{\prime \prime}\right) \mathfrak{S}_{5}
\end{aligned}
$$

and

$$
\begin{aligned}
& \partial_{i} r_{H}^{(1)}=\left(2 r^{3}+r^{5} f F^{\prime}\right)\left(\frac{1}{4} \mathbf{v}_{5}+\frac{5}{36} \mathfrak{V}_{1}-\frac{5}{24} \mathfrak{V}_{2}-\frac{11}{24} \mathfrak{V}_{3}\right) \\
& \partial_{0} r_{H}^{(1)}=\frac{1}{3 r}\left(\mathbf{s}_{2}+\mathbf{s}_{3}-\mathfrak{S}_{1}-\mathfrak{S}_{4}\right)+\left(\frac{1}{3 r}+\frac{1}{6} r^{3}+\frac{1}{12} r^{5} f F^{\prime}\right) \mathfrak{S}_{5}, \\
&\left(r^{4} k_{(2)}\right)^{\prime}+8 r^{3} j_{(2)}-\left(3 r^{4}-1\right) h_{(2)}^{\prime}=-\frac{4}{3} r \mathbf{s}_{2}+\frac{2}{3} r \mathfrak{S}_{1}-\frac{2}{9} r \mathfrak{S}_{3}+\left(\frac{1}{3 r^{3}}+2 r\right) \mathfrak{S}_{4} \\
&-\left[\frac{5}{3} r+\frac{1}{3}\left(3 r^{4}-1\right) F F^{\prime}+\frac{1}{12} r^{5} f F^{\prime 2}\right] \mathfrak{S}_{5} .
\end{aligned}
$$

\footnotetext{
${ }^{4}$ Please note that there is no EOM for dilaton in D3-brane case.
} 
During the procedure of casting the above differential equations, it needs to use the differential equations of first order perturbations like eqs. (3.12), (3.13), (3.14), (3.15), (3.18), together with the Navier-Stokes equations (5.16), (5.17) to eliminate the $x^{\mu}$ dependent terms.

Now we can use eqs. (5.18), (5.20) separately to get the solutions for the differential equations of tensor eq. (5.23) and vector eq. (5.24) perturbations:

$$
\begin{aligned}
& \alpha_{i j}^{(2)}=\frac{2-\ln 2}{4 r^{4}}\left(\mathbf{t}_{3}+\mathfrak{T}_{1}+\frac{\mathfrak{T}_{4}}{3}\right)+\frac{1}{r^{2}} \mathfrak{T}_{5}+\left(\frac{1}{r^{2}}+\frac{1}{2 r^{4}}\right) \mathfrak{T}_{6}+\left(\frac{2}{r^{2}}-\frac{\ln 2}{2 r^{4}}\right) \mathfrak{T}_{7}, \\
& w_{i}^{(2)}=\frac{1}{2 r^{2}}\left(\mathbf{v}_{4}+\mathbf{v}_{5}\right)+\frac{1}{9 r^{2}} \mathfrak{V}_{1}-\frac{2}{3 r^{2}}\left(\mathfrak{V}_{2}+\mathfrak{V}_{3}\right) .
\end{aligned}
$$

As for the scalar part, first we should use eq. (5.25) together with the gauge condition to get the differential equation for $h^{(2)}$, then solve it via

$$
h^{(2)}=\int_{r}^{\infty} \frac{1}{x^{5}} d x \int_{x}^{\infty} S_{h}(y) d y .
$$

As one can see from the above that the way to get $h^{(2)}$ for D3-brane is the same as $w_{i}$ eq. (5.20), the other Dp-branes will follow the way of eq. (5.18). Then one can get $j^{(2)}$ and $k^{(2)}$ easily from eq. (5.19), the results of the scalar perturbations for $p=3$ are

$$
\begin{aligned}
h^{(2)} & =-\frac{1}{6 r^{2}} \mathfrak{S}_{4}-\frac{1}{6 r^{2}} \mathfrak{S}_{5}, \\
j^{(2)} & =-\frac{3}{2} h^{(2)}=\frac{1}{4 r^{2}} \mathfrak{S}_{4}+\frac{1}{4 r^{2}} \mathfrak{S}_{5}, \\
k^{(2)} & =-\frac{2}{3 r^{2}} \mathbf{S}_{2}+\frac{1}{3 r^{2}} \mathfrak{S}_{1}-\frac{1}{9 r^{2}} \mathfrak{S}_{3}+\frac{1}{2 r^{2}} \mathfrak{S}_{4}+\frac{1}{2 r^{2}} \mathfrak{S}_{5} .
\end{aligned}
$$

We only retain the leading order in the large $r$ expansion in presenting the solutions of the vector and scalar perturbations. Because both of these two parts are trivial and only the leading order is relevant in calculating the stress-energy tensor of the dual fluid. The first scalar constraint eq. (5.27) and the vector constraint eq. (5.26) will reproduce the Navier-Stokes equations (5.16), (5.17) after we expand $F(r)$.

\subsection{The D4-brane case}

Solving the D4-brane is almost the same like the compactified D4-brane case [13], we just need to keep in mind that the spatial dimension is 4 but not 3 for D4-brane case here. The differential equations for tensor perturbation is

$$
\begin{aligned}
\partial_{r}\left(r^{4} f \partial_{r} \alpha_{i j}^{(2)}\right)= & \left(6 r-\frac{5}{2} r^{\frac{3}{2}} F-2 r^{\frac{5}{2}} F^{\prime}\right)\left(\mathbf{t}_{3}+\mathfrak{T}_{1}\right)+\left[2 r+2 r^{\frac{3}{2}} F-\frac{21}{5} r^{\frac{5}{2}} F^{\prime}\right. \\
& -\frac{4}{5} r^{\frac{7}{2}} F^{\prime \prime}+\left(4 r^{3}-1\right) F F^{\prime}+r^{4} f F F^{\prime \prime}+5 r^{\frac{3}{2}} F_{j}-15 r^{2} F F_{j} \\
& +2\left(4 r^{3}-1\right) F^{\prime} F_{j}-\left(5 r^{3}-2\right) F F_{j}^{\prime}+r^{4} f F^{\prime} F_{j}^{\prime}+2 r^{4} f F^{\prime \prime} F_{j} \\
& \left.-\frac{15}{2} r^{2} F F_{k}+4 r^{3} F^{\prime} F_{k}-\frac{13}{2} r^{3} F F_{k}^{\prime}+r^{4} F^{\prime} F_{k}^{\prime}+r^{4} F^{\prime \prime} F_{k}-r^{4} F F_{k}^{\prime \prime}\right] \mathfrak{T}_{4} \\
& -\left(\frac{4}{r^{2}}+8 r\right) \mathfrak{T}_{5}+\left(8 r-5 r^{\frac{3}{2}} F+r^{4} f F^{\prime 2}\right) \mathfrak{T}_{6} \\
& -\left(4 r+5 r^{\frac{3}{2}} F+4 r^{\frac{5}{2}} F^{\prime}\right) \mathfrak{T}_{7},
\end{aligned}
$$


Compared with the compactified D4-brane in [13], the above has the same coefficient functions for viscous tensors $\mathbf{t}_{3}, \mathfrak{T}_{1,5,6,7}$ (the definitions for $\mathfrak{T}_{5,6,7}$ are different from [13]). Only the coefficient function of $\mathfrak{T}_{4}$ is different. Since coefficient functions determine the transport coefficients. Thus confirms that $\tau_{\pi}, \lambda_{1,2}$ of D4-brane are the same as the compactified D4-brane but $\tau_{\pi}^{*}$ is different. The solution of eq. (5.33) can be got from eq. (5.18) as

$$
\begin{aligned}
\alpha_{i j}^{(2)}= & \left(\frac{4}{3}-\frac{\pi}{9 \sqrt{3}}-\frac{\ln 3}{3}\right) \frac{1}{r^{3}}\left(\mathbf{t}_{3}+\mathfrak{T}_{1}\right)+\left[\frac{2}{r}+\left(\frac{2}{5}-\frac{\pi}{45 \sqrt{3}}-\frac{\ln 3}{15}\right) \frac{1}{r^{3}}\right] \mathfrak{T}_{4} \\
& +\frac{4}{r} \mathfrak{T}_{5}+\left(\frac{4}{r}+\frac{4}{3 r^{3}}\right) \mathfrak{T}_{6}+\left[\frac{8}{r}-\left(\frac{2 \pi}{9 \sqrt{3}}+\frac{2 \ln 3}{3}\right) \frac{1}{r^{3}}\right] \mathfrak{T}_{7} .
\end{aligned}
$$

The differential equation for the 2nd order vector perturbation of D4-brane is

$$
\begin{aligned}
\partial_{r}\left(r^{4} \partial_{r} w_{i}^{(2)}\right)= & \left(-2 r-r^{\frac{5}{2}} F^{\prime}+\frac{10}{3} r^{\frac{3}{2}} F_{j}-\frac{4}{3} r^{\frac{5}{2}} F_{j}^{\prime}\right) \mathbf{v}_{4}+\left(\frac{10}{3} r^{\frac{3}{2}} F_{j}-\frac{4}{3} r^{\frac{5}{2}} F_{j}^{\prime}\right) \mathbf{v}_{5} \\
& +\left(\frac{3}{4} r-\frac{29}{8} r^{\frac{5}{2}} F^{\prime}-2 r^{\frac{7}{2}} F^{\prime \prime}+\frac{45}{8} r^{\frac{3}{2}} F_{j}+\frac{11}{4} r^{\frac{5}{2}} F_{j}^{\prime}-2 r^{\frac{7}{2}} F_{j}^{\prime \prime}\right) \mathfrak{V}_{1} \\
& +\left(5 r-\frac{3}{2} r^{\frac{5}{2}} F^{\prime}+\frac{5}{2} r^{\frac{3}{2}} F_{j}-r^{\frac{5}{2}} F_{j}^{\prime}\right) \mathfrak{V}_{2} \\
& +\left(-r-\frac{9}{2} r^{\frac{5}{2}} F^{\prime}+\frac{5}{2} r^{\frac{3}{2}} F_{j}-r^{\frac{5}{2}} F_{j}^{\prime}\right) \mathfrak{V}_{3},
\end{aligned}
$$

whose solution can be got from eq. (5.20) as

$$
w_{i}^{(2)}=-\frac{1}{r} \mathfrak{V}_{1}-\frac{4}{r} \mathfrak{V}_{2}-\frac{4}{r} \mathfrak{V}_{3}+\mathcal{O}\left(\frac{1}{r^{7 / 2}}\right) .
$$

Here we only keep the leading order for the vector perturbation, the same as D3-brane. The D1 and D2-brane will be treated similarly. The vector constraint is

$$
\begin{aligned}
\partial_{i} r_{H}^{(1)}= & {\left[-\frac{4}{3} r^{\frac{5}{2}}-\frac{2}{3} r^{4} f F^{\prime}+\frac{8}{9}\left(5 r^{3}-2\right) F_{j}+\frac{20}{9} r^{3} F_{k}+\frac{8}{9} r^{4} F_{k}^{\prime}\right] \mathbf{v}_{4} } \\
& +\left[\frac{8}{9}\left(5 r^{3}-2\right) F_{j}+\frac{20}{9} r^{3} F_{k}+\frac{8}{9} r^{4} F_{k}^{\prime}\right] \mathbf{v}_{5} \\
& +\left[\frac{35}{6} r^{\frac{5}{2}}-\frac{11}{12} r F^{\prime}+\frac{35}{12} r^{4} F^{\prime}-\frac{5}{3} F_{j}-\frac{95}{6} r^{3} F_{j}-\frac{95}{12} r^{3} F_{k}-\frac{5}{2} r^{4} F_{k}^{\prime}\right] \mathfrak{V}_{1} \\
& +\left[-2 r^{\frac{5}{2}}-r^{4} f F^{\prime}+\frac{2}{3}\left(5 r^{3}-2\right) F_{j}+\frac{5}{3} r^{3} F_{k}+\frac{2}{3} r^{4} F_{k}^{\prime}\right] \mathfrak{V}_{2} \\
& +\left[-6 r^{\frac{5}{2}}-3 r^{4} f F^{\prime}+\frac{2}{3}\left(5 r^{3}-2\right) F_{j}+\frac{5}{3} r^{3} F_{k}+\frac{2}{3} r^{4} F_{k}^{\prime}\right] \mathfrak{V}_{3} .
\end{aligned}
$$

After expanded, eq. (5.37) becomes

$$
\partial_{i} r_{H}^{(1)}=\frac{4}{45} \mathbf{v}_{4}+\frac{64}{45} \mathbf{v}_{5}+\frac{1}{2} \mathfrak{V}_{1}-\frac{14}{15} \mathfrak{V}_{2}-\frac{74}{15} \mathfrak{V}_{3},
$$

which is just eq. (5.17) with $p=4$. 
In the scalar sector, the first scalar perturbation

$$
\begin{aligned}
\partial_{0} r_{H}^{(1)}= & \left(\frac{4}{5 r^{\frac{1}{2}}}+\frac{4}{15} r^{\frac{5}{2}}-\frac{1}{5} F+\frac{2}{15} r^{4} f F^{\prime}-\frac{2}{3} r^{3} f F_{j}-\frac{1}{3} r^{3} F_{k}\right) \mathbf{s}_{2}+\frac{2}{5 r^{\frac{1}{2}}} \mathbf{s}_{3} \\
+ & \left(-\frac{8}{5 r^{\frac{1}{2}}}+\frac{4}{15} r^{\frac{5}{2}}-\frac{1}{5} F+\frac{2}{15} r^{4} f F^{\prime}-\frac{2}{3} r^{3} f F_{j}-\frac{1}{3} r^{3} F_{k}\right) \mathfrak{S}_{1} \\
+ & \left(\frac{1}{25 r^{\frac{1}{2}}}+\frac{1}{15} r^{\frac{5}{2}}-\frac{1}{25} F+\frac{17}{150} r^{4} f F^{\prime}+\frac{4}{75} r^{5} f F^{\prime \prime}-\frac{2}{15} r^{3} f F_{j}-\frac{4}{15} r^{4} f F_{j}^{\prime}\right. \\
& \left.\quad-\frac{1}{15} r^{3} F_{k}\right) \mathfrak{S}_{3}-\frac{4}{5 r^{\frac{1}{2}}} \mathfrak{S}_{4}+\left(\frac{4}{5 r^{\frac{1}{2}}}+\frac{4}{15} r^{\frac{5}{2}}+\frac{2}{15} r^{4} f F^{\prime}\right) \mathfrak{S}_{5}
\end{aligned}
$$

will reproduce the scalar component of Navier-Stokes equations eq. (5.16) with $p=4$. To get this, one needs to expand $F, F_{j}$ and $F_{k}$ in eq. (5.39) and has:

$$
\partial_{0} r_{H}^{(1)}=\frac{1}{75} \mathfrak{S}_{3}+\frac{4}{15} \mathfrak{S}_{5}+\frac{4}{5 r^{1 / 2}}\left(\mathbf{s}_{2}+\frac{1}{2} \mathbf{s}_{3}-2 \mathfrak{S}_{1}+\frac{1}{20} \mathfrak{S}_{3}-\mathfrak{S}_{4}+\mathfrak{S}_{5}\right) \text {. }
$$

The part inside the parenthesis is just the constraints eq. (5.11). Thus we reproduce eq. (5.16) for the case of $p=4$.

In order to solve $h, j, k$ at the second order, we use the second scalar perturbation eq. (3.11):

$$
\begin{aligned}
& 5\left(r^{3} k_{(2)}\right)^{\prime}+30 r^{2} j_{(2)}-4\left(5 r^{3}-2\right) h_{(2)}^{\prime}=\left(-16 r+5 r^{\frac{3}{2}} F+2 r^{\frac{5}{2}} F^{\prime}\right) \mathbf{s}_{2} \\
& +\left(24 r+5 r^{\frac{3}{2}} F+2 r^{\frac{5}{2}} F^{\prime}\right) \mathfrak{S}_{1}+\left[-\frac{5}{2} r+r^{\frac{5}{2}} F+\frac{26}{5} r^{\frac{5}{2}} F^{\prime}+\frac{4}{5} r^{\frac{7}{2}} F^{\prime \prime}+\frac{3}{8} r^{4} f F^{\prime 2}\right. \\
& -\frac{1}{4}\left(5 r^{3}-2\right) F F^{\prime}-10 r^{\frac{2}{3}} F_{j}+45 r^{2} F_{j}^{2}-2\left(5 r^{3}-2\right) F^{\prime} F_{j}-5 r^{3} F^{\prime} F_{k}-r^{4} F^{\prime} F_{k}^{\prime} \\
& \left.+30 r^{2} F_{j} F_{k}+10 r^{3} F_{j} F_{k}^{\prime}\right] \mathfrak{S}_{3}+\left(\frac{2}{r^{2}}+20 r\right) \mathfrak{S}_{4} \\
& -\left[18 r+\left(5 r^{3}-2\right) F F^{\prime}+\frac{1}{2} r^{4} f F^{\prime 2}\right] \mathfrak{S}_{5},
\end{aligned}
$$

the $(r r)$ component of Einstein equation (3.7):

$$
\begin{aligned}
4 r h_{(2)}^{\prime \prime}+6 h_{(2)}^{\prime}-5 j_{(2)}^{\prime}= & \left(\frac{1}{8} r F^{\prime 2}+\frac{3}{8} F F^{\prime}+\frac{1}{4} r F F^{\prime \prime}-5 F_{j} F_{j}^{\prime}+r F^{\prime} F_{j}^{\prime}\right) \mathfrak{S}_{3} \\
& +\frac{2}{r^{2}} \mathfrak{S}_{4}+\left(\frac{1}{2} r F^{\prime}+\frac{3}{2} F F^{\prime}+r F F^{\prime \prime}\right) \mathfrak{S}_{5}
\end{aligned}
$$

and the dilaton's EOM:

$$
\begin{aligned}
& \left(r^{3} k_{(2)}\right)^{\prime}+r^{3} f j_{(2)}^{\prime}+6 r^{2} j_{(2)}-2 r^{3} f h_{(2)}^{\prime}=\left(-2 r+\frac{1}{2} r^{\frac{3}{2}} F\right) \mathbf{s}_{2}+\left(6 r+\frac{1}{2} r^{\frac{3}{2}} F\right) \mathfrak{S}_{1} \\
& +\left[-\frac{1}{2} r+\frac{1}{10} r^{\frac{3}{2}} F+\frac{1}{5} r^{\frac{5}{2}} F^{\prime}-\frac{1}{8} r^{3} f F F^{\prime}-r^{\frac{3}{2}} F_{j}+9 r^{2} F_{j}^{2}+3 r^{3} f F_{j} F_{j}^{\prime}-r^{3} f F^{\prime} F_{j}\right. \\
& \left.-\frac{1}{2} r^{3} F^{\prime} F_{k}+6 r^{2} F_{j} F_{k}+r^{3} F_{j}^{\prime} F_{k}+2 r^{3} F_{j} F_{k}^{\prime}\right] \mathfrak{S}_{3}+2 r \mathfrak{S}_{4}-\left(2 r+\frac{1}{2} r^{3} f F F^{\prime}\right) \mathfrak{S}_{5}
\end{aligned}
$$


to eliminate $j$ and $k$ and get the different equation for $h_{(2)}$, then $h_{(2)}$ can be solved by using eq. (5.18). The final results for the 2 nd order scalar perturbations of D4-brane are

$$
\begin{aligned}
h^{(2)}= & \left(\frac{1}{3}-\frac{\pi}{36 \sqrt{3}}-\frac{\ln 3}{12}\right) \frac{1}{r^{3}}\left(\mathbf{s}_{2}+\mathfrak{S}_{1}\right)+\left[\frac{1}{4 r}+\left(\frac{1}{60}-\frac{\pi}{180 \sqrt{3}}-\frac{\ln 3}{60}\right) \frac{1}{r^{3}}\right] \mathfrak{S}_{3} \\
& +\frac{1}{r} \mathfrak{S}_{4}+\left(\frac{1}{r}+\frac{1}{3 r^{3}}\right) \mathfrak{S}_{5}, \\
j^{(2)}= & -\left(\frac{2}{3}-\frac{\pi}{18 \sqrt{3}}-\frac{\ln 3}{6}\right) \frac{1}{r^{3}}\left(\mathbf{s}_{2}+\mathfrak{S}_{1}\right)-\left(\frac{1}{30}-\frac{\pi}{90 \sqrt{3}}-\frac{\ln 3}{30}\right) \frac{1}{r^{3}} \mathfrak{S}_{3} \\
& -\frac{2}{3 r^{3}} \mathfrak{S}_{5}, \\
r^{3} k^{(2)}= & \left.-\left(\frac{4}{15}-\frac{\pi}{45 \sqrt{3}}-\frac{\ln 3}{15}\right)+\frac{1}{10 r}-\left(\frac{2}{3}-\frac{\pi}{18 \sqrt{3}}-\frac{\ln 3}{6}\right) \frac{1}{r^{3}}\right] \mathbf{s}_{2} \\
& +\left[4 r^{2}-\left(\frac{4}{15}-\frac{\pi}{45 \sqrt{3}}-\frac{\ln 3}{15}\right)+\frac{1}{10 r}-\left(\frac{2}{3}-\frac{\pi}{18 \sqrt{3}}-\frac{\ln 3}{6}\right) \frac{1}{r^{3}}\right] \mathfrak{S}_{1} \\
& +\left[-\left(\frac{1}{75}-\frac{\pi}{225 \sqrt{3}}-\frac{\ln 3}{75}\right)+\frac{2}{35 r^{1 / 2}}+\frac{163}{100 r}+\left(\frac{1}{90}+\frac{\pi}{90 \sqrt{3}}+\frac{\ln 3}{30}\right) \frac{1}{r^{3}}\right] \mathfrak{S}_{3} \\
& -\frac{2}{r} \mathfrak{S}_{4}-\left(\frac{4}{15}-\frac{8}{7 r^{1 / 2}}-\frac{1}{r}+\frac{2}{3 r^{3}}\right) \mathfrak{S}_{5} .
\end{aligned}
$$

Here the constants at the right hand side of the solution of $k^{(2)}$ are integration constants, which are fixed by requiring the boundary stress-energy tensor is in Landau frame. We do not need to fix the integration constants for D3-brane because the scalar perturbations are trivial and do not contribute to the stress-energy tensor.

\subsection{The D1-brane case}

The D1-brane case is very different from the other cases for no viscous tensors can exist in this case. We list the viscous scalars and vectors for $p=1$ in table 2 . Note we define a new viscous vector $\mathbf{v}_{3}=\partial_{1}^{2} \beta_{1}$ for D1-brane out of a linear combination of $\mathbf{v}_{4}$ and $\mathbf{v}_{5}$ since both of them can not exist for $p=1$. The viscous terms for D1-brane case satisfy the following 4 constraints:

$$
\begin{aligned}
\mathbf{s}_{1}+\frac{1}{4} \mathbf{s}_{2}-\frac{1}{4} \mathfrak{S}_{1}-\frac{1}{16} \mathfrak{S}_{3} & =0 \\
\mathbf{s}_{2}+2 \mathbf{s}_{3}-\frac{1}{2} \mathfrak{S}_{1}+\frac{1}{2} \mathfrak{S}_{3} & =0 \\
\mathbf{v}_{1}+\frac{1}{2} \mathbf{v}_{2}+\frac{1}{8} \mathfrak{V}_{1} & =0 \\
\mathbf{v}_{1}+\frac{1}{4} \mathbf{v}_{3}-\frac{3}{8} \mathfrak{V}_{1} & =0
\end{aligned}
$$

which can be got from the general form of the constraints eqs. (5.10), (5.12), (5.13), (5.11) by specifying $p=1$. 


\begin{tabular}{|l|l|}
\hline Scalars of $\mathrm{SO}(1)$ & Vectors of $\mathrm{SO}(1)$ \\
\hline $\mathbf{s}_{1}=\frac{1}{r_{H}} \partial_{0}^{2} r_{H}$ & $\mathbf{v}_{1}=\frac{1}{r_{H}} \partial_{0} \partial_{1} r_{H}$ \\
$\mathbf{s}_{2}=\partial_{0} \partial_{1} \beta_{1}$ & $\mathbf{v}_{2}=\partial_{0}^{2} \beta_{1}$ \\
$\mathbf{s}_{3}=\frac{1}{r_{H}} \partial_{1}^{2} r_{H}$ & $\mathbf{v}_{3}=\partial_{1}^{2} \beta_{1}$ \\
$\mathfrak{S}_{1}=\partial_{0} \beta_{1} \partial_{0} \beta_{1}$ & $\mathfrak{V}_{1}=\partial_{0} \beta_{1} \partial_{1} \beta_{1}$ \\
$\mathfrak{S}_{3}=\left(\partial_{1} \beta_{1}\right)^{2}$ & \\
\hline
\end{tabular}

Table 2. The list of all the second order viscous terms for D1-brane. The vector viscous terms are reduced to scalars in this case but we still call them vectors.

There is no tensor perturbation for D1-brane, thus we start from the vector perturbation. The vector constraint for D1-brane is

$$
\begin{aligned}
\partial_{1} r_{H}^{(1)}= & {\left[-\frac{1}{6} F_{j}+\frac{2}{3} r^{6} F_{j}+\frac{1}{3} r^{6} F_{k}+\frac{1}{12} r^{7} F_{k}^{\prime}\right] \mathbf{v}_{3} } \\
& +\left[\frac{1}{6} r^{4}+\frac{1}{24} r F^{\prime}+\frac{1}{12} r^{7} F^{\prime}-\frac{1}{3}\left(2 r^{6}+1\right) F_{j}-\frac{1}{3} r^{6} F_{k}\right] \mathfrak{V}_{1} .
\end{aligned}
$$

After expansion the above becomes

$$
\partial_{1} r_{H}^{(1)}=\frac{1}{12} \mathbf{v}_{3}
$$

which is just the spatial component of the Navier-Stokes equation (5.17). The vector dynamical equation is

$$
\partial_{r}\left(r^{7} \partial_{r} w_{1}^{(2)}\right)=\left(4 r^{3} F_{j}-r^{4} F_{j}^{\prime}\right) \mathbf{v}_{3}+\left(-\frac{5}{2} r^{4} F^{\prime}-\frac{1}{2} r^{5} F^{\prime \prime}+12 r^{3} F_{j}+\frac{1}{2} r^{4} F_{j}^{\prime}-\frac{1}{2} r^{5} F_{j}^{\prime \prime}\right) \mathfrak{V}_{1}
$$

from which we get

$$
w_{1}^{(2)}=-\frac{1}{4 r^{4}} \mathfrak{V}_{1} .
$$

The scalar constraint for D1-brane is

$$
\begin{aligned}
\partial_{0} r_{H}^{(1)}= & \left(\frac{1}{8 r^{2}}+\frac{1}{12} r^{4}-\frac{1}{8} F+\frac{1}{24} r^{7} f F^{\prime}-\frac{1}{3} r^{6} f F_{j}-\frac{1}{6} r^{6} F_{k}\right) \mathbf{s}_{2}+\frac{1}{4 r^{2}} \mathbf{s}_{3} \\
& +\left(-\frac{1}{16 r^{2}}+\frac{1}{12} r^{4}-\frac{1}{8} F+\frac{1}{24} r^{7} f F^{\prime}-\frac{1}{3} r^{6} f F_{j}-\frac{1}{6} r^{6} F_{k}\right) \mathfrak{S}_{1} \\
& +\left(\frac{1}{16 r^{2}}+\frac{1}{12} r^{4}-\frac{1}{16} F+\frac{7}{96} r^{7} f F^{\prime}+\frac{1}{96} r^{8} f F^{\prime \prime}-\frac{1}{6} r^{6} f F_{j}-\frac{1}{12} r^{7} f F_{j}^{\prime}\right. \\
& \left.\quad-\frac{1}{12} r^{6} F_{k}\right) \mathfrak{S}_{3}
\end{aligned}
$$

under the large $r$ expansion one has

$$
\partial_{0} r_{H}^{(1)}=\frac{1}{24} \mathfrak{S}_{3}+\frac{1}{8 r^{2}}\left(\mathbf{s}_{2}+2 \mathbf{s}_{3}-\frac{1}{2} \mathfrak{S}_{1}+\frac{1}{2} \mathfrak{S}_{3}\right)
$$


The terms inside the parenthesis in the above equation is just the 2 nd order constraint of D1-brane eq. (5.46) thus gives no contribution. So we get the scalar component of the Navier-Stokes equation for D1-brane as

$$
\partial_{0} r_{H}^{(1)}=\frac{1}{24} \mathfrak{S}_{3}
$$

which can be got from eq. (5.16) by setting $p=1$ and remove $\mathfrak{S}_{5}$ (there is no shear tensor for D1-brane). The dynamical equation for the scalar perturbations are the second scalar constraint eq. (3.11)

$$
\begin{aligned}
4\left(r^{6} k_{(2)}\right)^{\prime}+ & 48 r^{5} j_{(2)}-\left(4 r^{6}-1\right) h_{(2)}^{\prime} \\
=(-2 r+ & \left.4 r^{3} F+r^{4} F^{\prime}\right) \mathbf{s}_{2}+\left(4 r^{3} F+r^{4} F^{\prime}\right) \mathfrak{S}_{1}+\left[-2 r+2 r^{3} F+\frac{11}{4} r^{4} F^{\prime}+\frac{1}{4} r^{5} F^{\prime \prime}\right. \\
& -\left(4 r^{6}-1\right) F F^{\prime}-8 r^{3} F_{j}+72 r^{5} F_{j}^{2}-2\left(4 r^{6}-1\right) F^{\prime} F_{j}-4 r^{6} F^{\prime} F_{k}-\frac{1}{2} r^{7} F^{\prime} F_{k}^{\prime} \\
& \left.+48 r^{5} F_{j} F_{k}+8 r^{6} F_{j} F_{k}^{\prime}\right] \mathfrak{S}_{3},
\end{aligned}
$$

the $(r r)$ component of Einstein equation (3.7)

$$
r h_{(2)}^{\prime \prime}+3 h_{(2)}^{\prime}-8 j_{(2)}^{\prime}=\left(\frac{1}{2} r F^{2}+3 F F^{\prime}+r F F^{\prime \prime}-8 F_{j} F_{j}^{\prime}+r F^{\prime} F_{j}^{\prime}\right) \mathfrak{S}_{3},
$$

and the EOM of $\phi$ eq. (3.8)

$$
\begin{aligned}
2\left(r^{6} k_{(2)}\right)^{\prime}+ & 2 r^{6} f j_{(2)}^{\prime}+24 r^{5} j_{(2)}-r^{6} f h_{(2)}^{\prime}=\left(-r+r^{3} F\right) \mathbf{s}_{2}+r^{3} F \mathfrak{S}_{1} \\
+ & {\left[-r+\frac{1}{2} r^{3} F+\frac{1}{4} r^{4} F^{\prime}-r^{6} f F F^{\prime}-2 r^{3} F_{j}+36 r^{5} F_{j}^{2}+6 r^{6} f F_{j} F_{j}^{\prime}-2 r^{6} f F^{\prime} F_{j}\right.} \\
& \left.-r^{6} F^{\prime} F_{k}+24 r^{5} F_{j} F_{k}+2 r^{6} F_{j}^{\prime} F_{k}+4 r^{6} F_{j} F_{k}^{\prime}\right] \mathfrak{S}_{3},
\end{aligned}
$$

from which we can solve all the scalar perturbations as

$$
\begin{aligned}
h^{(2)}= & \left(\frac{1}{6}+\frac{\pi}{36 \sqrt{3}}-\frac{\ln 3}{12}\right) \frac{1}{r^{6}}\left(\mathbf{s}_{2}+\mathfrak{S}_{1}\right)+\left[\frac{1}{4 r^{4}}+\left(\frac{1}{12}+\frac{\pi}{72 \sqrt{3}}-\frac{\ln 3}{24}\right) \frac{1}{r^{6}}\right] \mathfrak{S}_{3}, \\
j^{(2)}=- & \left(\frac{1}{12}+\frac{\pi}{72 \sqrt{3}}-\frac{\ln 3}{24}\right) \frac{1}{r^{6}}\left(\mathbf{s}_{2}+\mathfrak{S}_{1}\right)-\left(\frac{1}{24}+\frac{\pi}{144 \sqrt{3}}-\frac{\ln 3}{48}\right) \frac{1}{r^{6}} \mathfrak{S}_{3}, \\
r^{6} k^{(2)}=- & {\left[\left(\frac{1}{12}+\frac{\pi}{72 \sqrt{3}}-\frac{\ln 3}{24}\right)-\frac{1}{40 r^{4}}+\left(\frac{1}{12}+\frac{\pi}{72 \sqrt{3}}-\frac{\ln 3}{24}\right) \frac{1}{r^{6}}\right] \mathbf{s}_{2} } \\
+ & {\left[\frac{1}{4} r^{2}-\left(\frac{1}{12}+\frac{\pi}{72 \sqrt{3}}-\frac{\ln 3}{24}\right)+\frac{1}{40 r^{4}}-\left(\frac{1}{12}+\frac{\pi}{72 \sqrt{3}}-\frac{\ln 3}{24}\right) \frac{1}{r^{6}}\right] \mathfrak{S}_{1} } \\
& -\left[\left(\frac{1}{24}+\frac{\pi}{144 \sqrt{3}}-\frac{\ln 3}{48}\right)-\frac{1}{16 r^{2}}-\frac{1}{16 r^{4}}\right. \\
& \left.+\left(\frac{1}{72}+\frac{\pi}{144 \sqrt{3}}-\frac{\ln 3}{48}\right) \frac{1}{r^{6}}\right] \mathfrak{S}_{3} .
\end{aligned}
$$




\subsection{The D2-brane case}

The D2-brane case is the most complicated one and that's why we leave it to the last. The differential equation for the tensor perturbation of D2-brane is

$$
\begin{aligned}
\partial_{r}\left(r^{6} f \partial_{r} \alpha_{i j}^{(2)}\right)=( & \left.\frac{2}{3} r-\frac{7}{2} r^{\frac{5}{2}} F-2 r^{\frac{7}{2}} F^{\prime}\right)\left(t_{3}+\mathfrak{T}_{1}\right)+\left[\frac{2}{3} r-\frac{3}{2} r^{\frac{5}{2}} F-\frac{31}{7} r^{\frac{7}{2}} F^{\prime}\right. \\
& -\frac{4}{7} r^{\frac{9}{2}} F^{\prime \prime}+\frac{1}{2} r^{6} f F^{\prime 2}+\left(6 r^{5}-1\right) F F^{\prime}+r^{6} f F F^{\prime \prime}+7 r^{\frac{5}{2}} F_{j}-35 r^{4} F F_{j} \\
& +2\left(6 r^{5}-1\right) F^{\prime} F_{j}+2 r^{6} f F^{\prime \prime} F_{j}-\left(7 r^{5}-2\right) F F_{j}^{\prime}+r^{6} f F^{\prime} F_{j}^{\prime}-\frac{35}{2} r^{4} F F_{k} \\
& \left.+6 r^{5} F^{\prime} F_{k}+r^{6} F^{\prime \prime} F_{k}-\frac{19}{2} r^{5} F F_{k}^{\prime}+r^{6} F^{\prime} F_{k}^{\prime}-r^{6} F F_{k}^{\prime \prime}\right] \mathfrak{T}_{4} \\
& -\left(4 r+7 r^{\frac{5}{2}} F+4 r^{\frac{7}{2}} F^{\prime}\right) \mathfrak{T}_{7} .
\end{aligned}
$$

Note in the source term there is no $\mathfrak{T}_{5,6}$ at present since both of them are zero for $p=2$. Using the relations between $F_{j, k}$ and $F$ eq. (3.29) and the differential equation for $F$ eq. (3.12) at the case $p=2$ will simplify the source term and make it easy to be integrated. The solution can be got from eq. (5.18) as

$$
\begin{aligned}
\alpha_{i j}^{(2)}(r)= & \left(\frac{4}{15}+\frac{\pi}{25} \sqrt{1-\frac{2}{\sqrt{5}}}+\frac{1}{5 \sqrt{5}} \operatorname{arcoth} \sqrt{5}-\frac{\ln 5}{10}\right) \frac{1}{r^{5}}\left(\mathbf{t}_{3}+\mathfrak{T}_{1}\right) \\
& +\left[\frac{4}{9 r^{3}}+\left(\frac{16}{105}+\frac{3 \pi}{175} \sqrt{1-\frac{2}{\sqrt{5}}}+\frac{3}{35 \sqrt{5}} \operatorname{arcoth} \sqrt{5}-\frac{3}{70} \ln 5\right) \frac{1}{r^{5}}\right] \mathfrak{T}_{4} \\
& +\left[\frac{8}{9 r^{3}}+\left(\frac{2 \pi}{25} \sqrt{1-\frac{2}{\sqrt{5}}}+\frac{2}{5 \sqrt{5}} \operatorname{arcoth} \sqrt{5}-\frac{\ln 5}{5}\right) \frac{1}{r^{5}}\right] \mathfrak{T}_{7} .
\end{aligned}
$$

As one can see from the above that the solution of D2-brane is indeed more complicated than the other nonconformal situations: except the terms proportional to $\pi$ and logarithm, there appears another arcoth term.

The 2nd order vector constraint for D2-brane is

$$
\begin{aligned}
\partial_{i} r_{H}^{(1)}= & {\left[-\frac{4}{15} r^{\frac{7}{2}}-\frac{2}{15} r^{6} f F^{\prime}+\frac{4}{15}\left(7 r^{5}-2\right) F_{j}+\frac{14}{15} r^{5} F_{k}+\frac{4}{15} r^{6} F_{k}^{\prime}\right] \mathbf{v}_{4} } \\
& +\left[\frac{4}{15}\left(7 r^{5}-2\right) F_{j}+\frac{14}{15} r^{5} F_{k}+\frac{4}{15} r^{6} F_{k}^{\prime}\right] \mathbf{v}_{5} \\
& +\left[\frac{23}{45} r^{\frac{7}{2}}-\frac{1}{30} r F^{\prime}+\frac{23}{90} r^{6} F^{\prime}-\frac{2}{5} F_{j}-\frac{77}{45} r^{5} F_{j}-\frac{77}{90} r^{5} F_{k}-\frac{1}{9} r^{6} F_{k}^{\prime}\right] \mathfrak{V}_{1} \\
& +\left[-\frac{2}{5} r^{\frac{7}{2}}-\frac{1}{5} r^{6} f F^{\prime}+\frac{2}{15}\left(7 r^{5}-2\right) F_{j}+\frac{7}{15} r^{5} F_{k}+\frac{2}{15} r^{6} F_{k}^{\prime}\right] \mathfrak{V}_{2} \\
& +\left[-\frac{22}{45} r^{\frac{7}{2}}-\frac{11}{45} r^{6} f F^{\prime}+\frac{2}{15}\left(7 r^{5}-2\right) F_{j}+\frac{7}{15} r^{5} F_{k}+\frac{2}{15} r^{6} F_{k}^{\prime}\right] \mathfrak{V}_{3} .
\end{aligned}
$$

After large $r$ expansion, the above becomes

$$
\partial_{i} r_{H}^{(1)}=\frac{4}{105} \mathbf{v}_{4}+\frac{32}{105} \mathbf{v}_{5}+\frac{37}{315} \mathfrak{V}_{1}-\frac{26}{105} \mathfrak{V}_{2}-\frac{106}{315} \mathfrak{V}_{3} .
$$


This is just the vector component of Navier-Stokes equation (5.17) when $p=2$. The vector dynamical equation is

$$
\begin{aligned}
\partial_{r}\left(r^{6} \partial_{r} w_{i}^{(2)}\right)= & \left(-2 r-r^{\frac{7}{2}} F^{\prime}+7 r^{\frac{5}{2}} F_{j}-2 r^{\frac{7}{2}} F_{j}^{\prime}\right) \mathbf{v}_{4}+\left(7 r^{\frac{5}{2}} F_{j}-2 r^{\frac{7}{2}} F_{j}^{\prime}\right) \mathbf{v}_{5} \\
& +\left(\frac{1}{2} r-\frac{25}{12} r^{\frac{7}{2}} F^{\prime}-\frac{2}{3} r^{\frac{9}{2}} F^{\prime \prime}+\frac{35}{4} r^{\frac{5}{2}} F_{j}+\frac{7}{6} r^{\frac{7}{2}} F_{j}^{\prime}-\frac{2}{3} r^{\frac{9}{2}} F_{j}^{\prime \prime}\right) \mathfrak{V}_{1} \\
& +\left(-\frac{1}{3} r-\frac{3}{2} r^{\frac{7}{2}} F^{\prime}+\frac{7}{2} r^{\frac{5}{2}} F_{j}-r^{\frac{7}{2}} F_{j}^{\prime}\right) \mathfrak{V}_{2} \\
& +\left(-r-\frac{11}{6} r^{\frac{7}{2}} F^{\prime}+\frac{7}{2} r^{\frac{5}{2}} F_{j}-r^{\frac{7}{2}} F_{j}^{\prime}\right) \mathfrak{V}_{3},
\end{aligned}
$$

whose leading order solution is

$$
w_{i}^{(2)}=-\frac{2}{9 r^{3}} \mathfrak{V}_{1}-\frac{4}{9 r^{3}} \mathfrak{V}_{2}-\frac{4}{9 r^{3}} \mathfrak{V}_{3} .
$$

The first scalar constraint for $p=2$ is

$$
\begin{aligned}
\partial_{0} r_{H}^{(1)}= & \left(\frac{4}{21 r^{3 / 2}}+\frac{4}{35} r^{\frac{7}{2}}-\frac{1}{7} F+\frac{2}{35} r^{6} f F^{\prime}-\frac{2}{5} r^{5} f F_{j}-\frac{1}{5} r^{5} F_{k}\right) \mathbf{s}_{2}+\frac{2}{7 r^{3 / 2}} \mathbf{s}_{3} \\
+ & \left(-\frac{8}{63 r^{3 / 2}}+\frac{4}{35} r^{\frac{7}{2}}-\frac{1}{7} F+\frac{2}{35} r^{6} f F^{\prime}-\frac{2}{5} r^{5} f F_{j}-\frac{1}{5} r^{5} F_{k}\right) \mathfrak{S}_{1} \\
+ & \left(\frac{2}{147 r^{3 / 2}}+\frac{2}{35} r^{\frac{7}{2}}-\frac{3}{49} F+\frac{17}{245} r^{6} f F^{\prime}+\frac{4}{245} r^{7} f F^{\prime \prime}-\frac{6}{35} r^{5} f F_{j}-\frac{4}{35} r^{6} f F_{j}^{\prime}\right. \\
& \left.\quad-\frac{3}{35} r^{5} F_{k}\right) \mathfrak{S}_{3}-\frac{4}{21 r^{3 / 2}} \mathfrak{S}_{4}+\left(\frac{4}{21 r^{3 / 2}}+\frac{4}{35} r^{\frac{7}{2}}+\frac{2}{35} r^{6} f F^{\prime}\right) \mathfrak{S}_{5} .
\end{aligned}
$$

After expansion, the above will reproduce the scalar component of the Navier-Stokes equation (5.16) for $p=2$ :

$$
\partial_{0} r_{H}^{(1)}=\frac{2}{245} \mathfrak{S}_{3}+\frac{4}{35} \mathfrak{S}_{5}+\frac{4}{21 r^{\frac{3}{2}}}\left(\mathbf{s}_{2}+\frac{3}{2} \mathbf{s}_{3}-\frac{2}{3} \mathfrak{S}_{1}+\frac{1}{14} \mathfrak{S}_{3}-\mathfrak{S}_{4}+\mathfrak{S}_{5}\right) .
$$

The terms in the parenthesis of the above is just the second order constraint eq. (5.11) at $p=2$ thus gives 0 . So we finally have

$$
\partial_{0} r_{H}^{(1)}=\frac{2}{245} \mathfrak{S}_{3}+\frac{4}{35} \mathfrak{S}_{5} .
$$

The above equation is just the scalar component of Navier-Stokes equation at $p=2$.

The scalar dynamical equations are still the second scalar constraint eq. (3.11)

$$
\begin{aligned}
7\left(r^{5} k_{(2)}\right)^{\prime} & +70 r^{4} j_{(2)}-2\left(7 r^{5}-2\right) h_{(2)}^{\prime} \\
= & \left(-\frac{16}{3} r+7 r^{\frac{5}{2}} F+2 r^{\frac{7}{2}} F^{\prime}\right) \mathbf{s}_{2}+\left(\frac{8}{9} r+7 r^{\frac{5}{2}} F+2 r^{\frac{7}{2}} F^{\prime}\right) \mathfrak{S}_{1}+\left[-\frac{5}{3} r+3 r^{\frac{5}{2}} F\right. \\
& +\frac{38}{7} r^{\frac{7}{2}} F^{\prime}+\frac{4}{7} r^{\frac{9}{2}} F^{\prime \prime}+\frac{1}{4} r^{6} f F^{\prime 2}-\frac{1}{2}\left(7 r^{5}-2\right) F F^{\prime}-14 r^{\frac{5}{2}} F_{j}+105 r^{4} F_{j}^{2} \\
& \left.\quad 2\left(7 r^{5}-2\right) F^{\prime} F_{j}-7 r^{5} F^{\prime} F_{k}-r^{6} F^{\prime} F_{k}^{\prime}+70 r^{4} F_{j} F_{k}+14 r^{5} F_{j} F_{k}^{\prime}\right] \mathfrak{S}_{3} \\
+ & \left(\frac{2}{r^{4}}+\frac{28}{3} r\right) \mathfrak{S}_{4}-\left[\frac{22}{3} r+\frac{1}{2} r^{6} f F^{\prime 2}+\left(7 r^{5}-2\right) F F^{\prime}\right] \mathfrak{S}_{5},
\end{aligned}
$$


the $(r r)$ component of the Einstein equation (3.7)

$$
\begin{aligned}
2 r h_{(2)}^{\prime \prime}+5 h_{(2)}^{\prime}-7 j_{(2)}^{\prime}= & \left(\frac{1}{4} r F^{\prime 2}+\frac{5}{4} F F^{\prime}+\frac{1}{2} r F F^{\prime \prime}-7 F_{j} F_{j}^{\prime}+r F^{\prime} F_{j}^{\prime}\right) \mathfrak{S}_{3} \\
& +\frac{2}{r^{4}} \mathfrak{S}_{4}+\left(\frac{1}{2} r F^{\prime 2}+\frac{5}{2} F F^{\prime}+r F F^{\prime \prime}\right) \mathfrak{S}_{5}
\end{aligned}
$$

and the EOM of dilaton eq. (3.8)

$$
\begin{aligned}
\left(r^{5} k_{(2)}\right)^{\prime}+r^{5} f j_{(2)}^{\prime}+10 r^{4} j_{(2)}-r^{5} f h_{(2)}^{\prime}=\left(-\frac{2}{3} r+\frac{1}{2} r^{\frac{5}{2}} F\right) \mathbf{s}_{2} \\
+\left(\frac{2}{9} r+\frac{1}{2} r^{\frac{5}{2}} F\right) \mathfrak{S}_{1}+\left[-\frac{1}{3} r+\frac{3}{14} r^{\frac{5}{2}} F+\frac{1}{7} r^{\frac{7}{2}} F^{\prime}-\frac{1}{4} r^{5} f F F^{\prime}-r^{\frac{5}{2}} F_{j}+15 r^{4} F_{j}^{2}\right. \\
\left.\quad-r^{5} f F^{\prime} F_{j}+3 r^{5} f F_{j} F_{j}^{\prime}-\frac{1}{2} r^{5} F^{\prime} F_{k}+10 r^{4} F_{j} F_{k}+r^{5} F_{j}^{\prime} F_{k}+2 r^{5} F_{j} F_{k}^{\prime}\right] \mathfrak{S}_{3} \\
\quad+\frac{2}{3} r \mathfrak{S}_{4}-\left(\frac{2}{3} r+\frac{1}{2} r^{5} f F F^{\prime}\right) \mathfrak{S}_{5} .
\end{aligned}
$$

From which we can solve the scalar perturbations for $p=2$ as

$$
\begin{aligned}
& h^{(2)}=\left(\frac{2}{15}+\frac{\pi}{50} \sqrt{1-\frac{2}{\sqrt{5}}}+\frac{1}{10 \sqrt{5}} \operatorname{arcoth} \sqrt{5}-\frac{\ln 5}{20}\right) \frac{1}{r^{5}}\left(\mathbf{s}_{2}+\mathfrak{S}_{1}\right) \\
& +\left[\frac{1}{9 r^{3}}+\left(\frac{1}{105}+\frac{3 \pi}{350} \sqrt{1-\frac{2}{\sqrt{5}}}+\frac{3}{70 \sqrt{5}} \operatorname{arcoth} \sqrt{5}-\frac{3}{140} \ln 5\right) \frac{1}{r^{5}}\right] \mathfrak{S}_{3} \\
& +\frac{2}{9 r^{3}} \mathfrak{S}_{4}+\left(\frac{2}{9 r^{3}}+\frac{2}{15 r^{5}}\right) \mathfrak{S}_{5} \\
& j^{(2)}=-\left(\frac{2}{15}+\frac{\pi}{50} \sqrt{1-\frac{2}{\sqrt{5}}}+\frac{1}{10 \sqrt{5}} \operatorname{arcoth} \sqrt{5}-\frac{\ln 5}{20}\right) \frac{1}{r^{5}}\left(\mathbf{s}_{2}+\mathfrak{S}_{1}\right) \\
& -\left(\frac{1}{105}+\frac{3 \pi}{350} \sqrt{1-\frac{2}{\sqrt{5}}}+\frac{3}{70 \sqrt{5}} \operatorname{arcoth} \sqrt{5}-\frac{\ln 5}{140}\right) \frac{1}{r^{5}} \mathfrak{S}_{3}-\frac{2}{15 r^{5}} \mathfrak{S}_{5}, \\
& r^{5} k^{(2)}=\left[-\left(\frac{4}{35}+\frac{3 \pi}{175} \sqrt{1-\frac{2}{\sqrt{5}}}+\frac{3}{35 \sqrt{5}} \operatorname{arcoth} \sqrt{5}-\frac{3}{70} \ln 5\right)+\frac{1}{28 r^{3}}\right. \\
& \left.-\left(\frac{2}{15}+\frac{\pi}{50} \sqrt{1-\frac{2}{\sqrt{5}}}+\frac{1}{10 \sqrt{5}} \operatorname{arcoth} \sqrt{5}-\frac{\ln 5}{20}\right) \frac{1}{r^{5}}\right] \mathbf{s}_{2} \\
& +\left[\frac{4}{9} r^{2}-\left(\frac{4}{35}+\frac{3 \pi}{175} \sqrt{1-\frac{2}{\sqrt{5}}}+\frac{3}{35 \sqrt{5}} \operatorname{arcoth} \sqrt{5}-\frac{3}{70} \ln 5\right)+\frac{1}{28 r^{3}}\right. \\
& \left.-\left(\frac{2}{15}+\frac{\pi}{50} \sqrt{1-\frac{2}{\sqrt{5}}}+\frac{1}{10 \sqrt{5}} \operatorname{arcoth} \sqrt{5}-\frac{\ln 5}{20}\right) \frac{1}{r^{5}}\right] \mathfrak{S}_{1} \\
& +\left[-\left(\frac{2}{245}+\frac{9 \pi}{1225} \sqrt{1-\frac{2}{\sqrt{5}}}+\frac{9}{245 \sqrt{5}} \operatorname{arcoth} \sqrt{5}-\frac{9}{490} \ln 5\right)+\frac{4}{273 r^{\frac{3}{2}}}\right.
\end{aligned}
$$




$$
\begin{aligned}
& \left.+\frac{17}{147 r^{3}}+\left(\frac{13}{525}-\frac{3 \pi}{350} \sqrt{1-\frac{2}{\sqrt{5}}}-\frac{3}{70 \sqrt{5}} \operatorname{arcoth} \sqrt{5}+\frac{3}{140} \ln 5\right) \frac{1}{r^{5}}\right] \mathfrak{S}_{3} \\
- & \frac{2}{9 r^{3}} \mathfrak{S}_{4}+\left(-\frac{4}{35}+\frac{8}{39 r^{\frac{3}{2}}}+\frac{1}{18 r^{3}}-\frac{2}{15 r^{5}}\right) \mathfrak{S}_{5} .
\end{aligned}
$$

\section{The second order stress-energy tensor}

\subsection{Formulation of the result}

Now, using the second order expanded metric eq. (5.3) and eq. (5.22) together with the already solved second order perturbations of Dp-brane, we can calculate the boundary stress-energy tensor via eq. (4.1). We will only offer the results here, the readers who are interested in the details can refer to [13].

In order to get the covariant form of the stress-energy tensor, we will need the following rules to substitute the 2 nd order spatial viscous tensors:

$$
\begin{array}{ll}
\mathbf{t}_{3 i j}=\partial_{0} \sigma_{i j} \rightarrow\left\langle D \partial_{\mu} u_{\nu\rangle},\right. & \mathfrak{T}_{1 i j}=\partial_{0} \beta_{i} \partial_{0} \beta_{j}-\frac{1}{p} \delta_{i j} \mathbf{s}_{1} \rightarrow D u_{\langle\mu} D u_{\nu\rangle}, \\
\mathfrak{T}_{4 i j}=\sigma_{i j} \partial \beta \rightarrow \sigma_{\mu \nu} \partial_{\rho} u^{\rho}, & \mathfrak{T}_{6 i j}=\sigma_{i k} \sigma_{k j}-\frac{1}{p} \delta_{i j} \mathbf{s}_{5} \rightarrow \sigma_{\langle\mu}{ }^{\rho} \sigma_{\nu\rangle \rho}, \\
\mathfrak{T}_{7 i j}=\sigma_{(i}{ }^{k} \Omega_{j) k} \rightarrow \sigma_{\langle\mu}{ }^{\rho} \Omega_{\nu\rangle \rho} &
\end{array}
$$

Then the boundary stress-energy tensor for D3-brane under full consideration of dimension can be calculated as

$$
\begin{aligned}
T_{\mu \nu}=\frac{1}{2 \kappa_{5}^{2}}\{ & \frac{r_{H}^{4}}{L_{3}^{5}}\left(3 u_{\mu} u_{\nu}+P_{\mu \nu}\right)-\left(\frac{r_{H}}{L_{3}}\right)^{3} \cdot 2 \sigma_{\mu \nu} \\
& \quad+\frac{r_{H}^{2}}{L_{3}}\left[\frac{2-\ln 2}{2} \cdot 2\left(\left\langle D \sigma_{\mu \nu\rangle}+\frac{1}{3} \sigma_{\mu \nu} \partial u\right)+\frac{1}{2} \cdot 4 \sigma_{\langle\mu}{ }^{\rho} \sigma_{\nu\rangle \rho}-\ln 2 \cdot 2 \sigma_{\langle\mu}{ }^{\rho} \Omega_{\nu\rangle \rho}\right]\right\} .
\end{aligned}
$$

Compare the above with eq. (1.1) one can get the 2nd order transport coefficients for D3-brane

$$
\eta \tau_{\pi}=\frac{1}{2 \kappa_{5}^{2}} \frac{2-\ln 2}{2} \frac{r_{H}^{2}}{L_{3}}, \quad \lambda_{1}=\frac{1}{2 \kappa_{5}^{2}} \frac{1}{2} \frac{r_{H}^{2}}{L_{3}}, \quad \lambda_{2}=-\frac{1}{2 \kappa_{5}^{2}} \ln 2 \frac{r_{H}^{2}}{L_{3}}
$$

We have restored the parameters $r_{H}, L_{p}$ and $\kappa_{p+2}^{2}$ which has length dimension in eqs. (6.2), (6.3). The results in eq. (6.3) have already been derived in e.g. [4, 6, 7].

As for the D4-brane, we need further substitution rules for the scalar viscous terms

$$
\mathbf{s}_{2}+\mathfrak{S}_{1}=\partial_{0} \partial \beta+\partial_{0} \beta_{i} \partial_{0} \beta_{i} \rightarrow D(\partial u), \mathfrak{S}_{3}=(\partial \beta)^{2} \rightarrow(\partial u)^{2}, \quad \mathfrak{S}_{5}=\sigma_{i j}^{2} \rightarrow \sigma_{\mu \nu}^{2}
$$


The boundary stress-energy tensor for D4-brane can then be formulated as

$$
\begin{aligned}
T_{\mu \nu}= & \frac{1}{2 \kappa_{6}^{2}}\left\{\frac{r_{H}^{3}}{L_{4}^{4}}\left(\frac{5}{2} u_{\mu} u_{\nu}+\frac{1}{2} P_{\mu \nu}\right)-\left(\frac{r_{H}}{L_{4}}\right)^{\frac{5}{2}}\left(2 \sigma_{\mu \nu}+\frac{1}{10} \partial_{\rho} u^{\rho} P_{\mu \nu}\right)\right. \\
& +\frac{r_{H}^{2}}{L_{4}}\left[( 2 - \frac { \pi } { 6 \sqrt { 3 } } - \frac { \operatorname { l n } 3 } { 2 } ) \cdot 2 \left(\left\langle D \sigma_{\mu \nu\rangle}+\frac{1}{4} \sigma_{\mu \nu} \partial u\right)+\left(\frac{2}{5}+\frac{\pi}{30 \sqrt{3}}+\frac{\ln 3}{10}\right) \frac{2 \sigma_{\mu \nu} \partial u}{4}\right.\right. \\
& \left.+4 \sigma_{\langle\mu}{ }^{\rho} \sigma_{\nu\rangle \rho}-\left(\frac{\pi}{3 \sqrt{3}}+\ln 3\right) \cdot 2 \sigma_{\langle\mu}{ }^{\rho} \Omega_{\nu\rangle \rho}\right]+\frac{r_{H}^{2}}{L_{4}} P_{\mu \nu}\left[\left(\frac{1}{5}-\frac{\pi}{60 \sqrt{3}}-\frac{\ln 3}{20}\right) D(\partial u)\right. \\
& \left.\left.+\left(\frac{1}{100}-\frac{\pi}{300 \sqrt{3}}-\frac{\ln 3}{100}\right)(\partial u)^{2}+\frac{1}{20} \cdot 4 \sigma_{\alpha \beta}^{2}\right]\right\}
\end{aligned}
$$

from which we can read all the second order transport coefficients as

$$
\begin{aligned}
\eta \tau_{\pi} & =\frac{1}{2 \kappa_{6}^{2}}\left(2-\frac{\pi}{6 \sqrt{3}}-\frac{\ln 3}{2}\right) \frac{r_{H}^{2}}{L_{4}}, \eta \tau_{\pi}^{*}=\frac{1}{2 \kappa_{6}^{2}}\left(\frac{2}{5}+\frac{\pi}{30 \sqrt{3}}+\frac{\ln 3}{10}\right) \frac{r_{H}^{2}}{L_{4}}, \lambda_{1}=\frac{1}{2 \kappa_{6}^{2}} \frac{r_{H}^{2}}{L_{4}} \\
\lambda_{2} & =-\frac{1}{2 \kappa_{6}^{2}}\left(\frac{\pi}{3 \sqrt{3}}+\ln 3\right) \frac{r_{H}^{2}}{L_{4}}, \zeta \tau_{\Pi}=\frac{1}{2 \kappa_{6}^{2}}\left(\frac{1}{5}-\frac{\pi}{60 \sqrt{3}}-\frac{\ln 3}{20}\right) \frac{r_{H}^{2}}{L_{4}}, \xi_{1}=\frac{1}{2 \kappa_{6}^{2}} \frac{1}{20} \frac{r_{H}^{2}}{L_{4}} \\
\xi_{2} & =\frac{1}{2 \kappa_{6}^{2}}\left(\frac{1}{100}-\frac{\pi}{300 \sqrt{3}}-\frac{\ln 3}{100}\right) \frac{r_{H}^{2}}{L_{4}} .
\end{aligned}
$$

Compared with the results for compactified D4-brane [13], one can see that $\eta \tau_{\pi}$ and $\lambda_{1,2}$ which are the transport coefficients of viscous tensors do indeed not change, just as what we have discussed in subsections 3.2, 5.3. But the other 4 coefficients i.e., $\eta \tau_{\pi}^{*}, \zeta \tau_{\Pi}$ and $\xi_{1,2}$ which associate with nonconformal viscous terms are different from that in [13]. This may lie in the difference of spatial dimensions. Further more, $\eta \tau_{\pi}^{*}$ and $\xi_{1}$ are separately half and $\frac{3}{8}$ of that for compactified D4-brane, while for $\zeta \tau_{\Pi}$ and $\xi_{2}$ there are no simple ratios.

As one can check, the shear and bulk relaxation time of D4-brane eq. (6.6) that we get here satisfies the relation in equation (4.7) of [16] after using the Hawking temperature eq. (4.4) with $p=4$.

The relativistic fluid dual to D1-brane has the stress-energy tensor

$$
\begin{aligned}
T_{\mu \nu}=\frac{1}{2 \kappa_{3}^{2}}\{ & \frac{r_{H}^{6}}{L_{1}^{7}}\left(4 u_{\mu} u_{\nu}+2 P_{\mu \nu}\right)-\left(\frac{r_{H}}{L_{1}}\right)^{4} P_{\mu \nu} \partial u \\
& \left.+\frac{r_{H}^{2}}{L_{1}} P_{\mu \nu}\left[\left(\frac{1}{2}+\frac{\pi}{12 \sqrt{3}}-\frac{\ln 3}{4}\right) D(\partial u)+\left(\frac{1}{4}+\frac{\pi}{24 \sqrt{3}}-\frac{\ln 3}{8}\right)(\partial u)^{2}\right]\right\}
\end{aligned}
$$

It has only two 2nd order transport coefficients from the scalar viscous terms

$$
\zeta \tau_{\Pi}=\frac{1}{2 \kappa_{3}^{2}}\left(\frac{1}{2}+\frac{\pi}{12 \sqrt{3}}-\frac{\ln 3}{4}\right) \frac{r_{H}^{2}}{L_{1}}, \quad \xi_{2}=\frac{1}{2 \kappa_{3}^{2}}\left(\frac{1}{4}+\frac{\pi}{24 \sqrt{3}}-\frac{\ln 3}{8}\right) \frac{r_{H}^{2}}{L_{1}} .
$$

One can check that our results of the 2nd order transport coefficients of D1-brane eq. (6.8) agree with equation (4.6) of [16] where the author derived the bulk relaxation time $\tau_{\Pi}$ for D1-brane. 
The stress-energy tensor of D2-brane is

$$
\begin{aligned}
& T_{\mu \nu}=\frac{1}{2 \kappa_{4}^{2}}\left\{\frac{r_{H}^{5}}{L_{2}^{6}}\left(\frac{7}{2} u_{\mu} u_{\nu}+\frac{3}{2} P_{\mu \nu}\right)-\left(\frac{r_{H}}{L_{2}}\right)^{\frac{7}{2}}\left(2 \sigma_{\mu \nu}+\frac{1}{7} P_{\mu \nu} \partial u\right)\right. \\
& +\frac{r_{H}^{2}}{L_{2}}\left[( \frac { 2 } { 3 } + \frac { \pi } { 1 0 } \sqrt { 1 - \frac { 2 } { \sqrt { 5 } } } + \frac { 1 } { 2 \sqrt { 5 } } \operatorname { a r c o t h } \sqrt { 5 } - \frac { \operatorname { l n } 5 } { 4 } ) 2 \left(\left\langle D \sigma_{\mu \nu\rangle}+\frac{1}{2} \sigma_{\mu \nu} \partial_{\rho} u^{\rho}\right)\right.\right. \\
& +\left(\frac{2}{21}-\frac{\pi}{70} \sqrt{1-\frac{2}{\sqrt{5}}}-\frac{1}{14 \sqrt{5}} \operatorname{arcoth} \sqrt{5}+\frac{\ln 5}{28}\right) 2 \cdot \frac{\sigma_{\mu \nu} \partial_{\rho} u^{\rho}}{2} \\
& \left.+\left(\frac{\pi}{5} \sqrt{1-\frac{2}{\sqrt{5}}}+\frac{1}{\sqrt{5}} \operatorname{arcoth} \sqrt{5}-\frac{\ln 5}{2}\right) 2 \sigma_{\langle\mu}{ }^{\rho} \Omega_{\nu\rangle \rho}\right] \\
& +\frac{r_{H}^{2}}{L_{2}} P_{\mu \nu}\left[\left(\frac{2}{21}+\frac{\pi}{70} \sqrt{1-\frac{2}{\sqrt{5}}}+\frac{1}{14 \sqrt{5}} \operatorname{arcoth} \sqrt{5}-\frac{\ln 5}{28}\right) D \partial u\right. \\
& \left.\left.+\left(\frac{1}{147}+\frac{3 \pi}{490} \sqrt{1-\frac{2}{\sqrt{5}}}+\frac{3}{98 \sqrt{5}} \operatorname{arcoth} \sqrt{5}-\frac{3 \ln 5}{196}\right)(\partial u)^{2}+\frac{1}{42} \cdot 4 \sigma_{\alpha \beta}^{2}\right]\right\} .
\end{aligned}
$$

The 2nd order transport coefficients can be read from the above as

$$
\begin{aligned}
& \eta \tau_{\pi}=\frac{1}{2 \kappa_{4}^{2}}\left(\frac{2}{3}+\frac{\pi}{10} \sqrt{1-\frac{2}{\sqrt{5}}}+\frac{1}{2 \sqrt{5}} \operatorname{arcoth} \sqrt{5}-\frac{\ln 5}{4}\right) \frac{r_{H}^{2}}{L_{2}}, \\
& \eta \tau_{\pi}^{*}=\frac{1}{2 \kappa_{4}^{2}}\left(\frac{2}{21}-\frac{\pi}{70} \sqrt{1-\frac{2}{\sqrt{5}}}-\frac{1}{14 \sqrt{5}} \operatorname{arcoth} \sqrt{5}+\frac{\ln 5}{28}\right) \frac{r_{H}^{2}}{L_{2}}, \\
& \lambda_{2}=\frac{1}{2 \kappa_{4}^{2}}\left(\frac{\pi}{5} \sqrt{1-\frac{2}{\sqrt{5}}}+\frac{1}{\sqrt{5}} \operatorname{arcoth} \sqrt{5}-\frac{\ln 5}{2}\right) \frac{r_{H}^{2}}{L_{2}}, \\
& \zeta \tau_{\Pi}=\frac{1}{2 \kappa_{4}^{2}}\left(\frac{2}{21}+\frac{\pi}{70} \sqrt{1-\frac{2}{\sqrt{5}}}+\frac{1}{14 \sqrt{5}} \operatorname{arcoth} \sqrt{5}-\frac{\ln 5}{28}\right) \frac{r_{H}^{2}}{L_{2}}, \quad \xi_{1}=\frac{1}{2 \kappa_{4}^{2}} \frac{1}{42} \frac{r_{H}^{2}}{L_{2}}, \\
& \xi_{2}=\frac{1}{2 \kappa_{4}^{2}}\left(\frac{1}{147}+\frac{3 \pi}{490} \sqrt{1-\frac{2}{\sqrt{5}}}+\frac{3}{98 \sqrt{5}} \operatorname{arcoth} \sqrt{5}-\frac{3}{196} \ln 5\right) \frac{r_{H}^{2}}{L_{2}} .
\end{aligned}
$$

Compared with the 2nd order coefficients of D4-brane, the D2-brane does not have $\lambda_{1}$. This is due to the fact that $\sigma_{\langle\mu}{ }^{\rho} \sigma_{\nu\rangle \rho}$ can not exist in $3 \mathrm{~d}$ relativistic hydrodynamics. In the literature, [47] also gives the explicit result of the shear relaxation time of D2-brane (as in equation (4.6) of [47]). But by comparing, we find that the shear relaxation time of D2-brane of our results eq. (6.10) does not agree with that in [47]. We think that the result offered in [47] should be wrong which will be specified later.

The 2 nd order stress-energy tensor for Dp-brane with $1 \leq p \leq 4$ can be nicely written in the following unified form in terms of Harmonic number:

$$
T_{\mu \nu}=\frac{1}{2 \kappa_{p+2}^{2}}\left\{\frac{r_{H}^{7-p}}{L_{p}^{8-p}}\left(\frac{9-p}{2} u_{\mu} u_{\nu}+\frac{5-p}{2} P_{\mu \nu}\right)-\left(\frac{r_{H}}{L_{p}}\right)^{\frac{9-p}{2}}\left(2 \sigma_{\mu \nu}+\frac{2(p-3)^{2}}{p(9-p)} P_{\mu \nu} \partial u\right)\right.
$$




$$
\begin{aligned}
+\frac{r_{H}^{2}}{L_{p}}[( & \left.\frac{1}{5-p}+\frac{1}{7-p} H_{\frac{5-p}{7-p}}\right) \cdot 2\left(\left\langle D \sigma_{\mu \nu\rangle}+\frac{1}{p} \sigma_{\mu \nu} \partial u\right)\right. \\
+ & \left(\frac{3(p-3)^{2}}{(5-p)(9-p)}-\frac{(p-3)^{2}}{(7-p)(9-p)} H_{\frac{5-p}{7-p}}\right) \frac{2 \sigma_{\mu \nu} \partial u}{p}+\frac{1}{5-p} \cdot 4 \sigma_{\langle\mu}{ }^{\rho} \sigma_{\nu\rangle \rho} \\
+ & \left.\left(-\frac{2}{5-p}+\frac{2}{7-p} H_{\frac{5-p}{7-p}}\right) \cdot 2 \sigma_{\langle\mu}{ }^{\rho} \Omega_{\nu\rangle \rho}\right] \\
+\frac{r_{H}^{2}}{L_{p}} P_{\mu \nu} & {\left[\left(\frac{2(p-3)^{2}}{p(5-p)(9-p)}+\frac{2(p-3)^{2}}{p(7-p)(9-p)} H_{\frac{5-p}{7-p}}\right) D(\partial u)\right.} \\
+ & \left(\frac{2(p-3)^{2}\left(3 p^{2}-17 p+18\right)}{p^{2}(5-p)(9-p)^{2}}+\frac{2(p-3)^{2}(5-p)}{p(7-p)(9-p)^{2}} H_{\frac{5-p}{7-p}}\right)(\partial u)^{2} \\
+ & \left.\left.\frac{(p-3)^{2}}{p(5-p)(9-p)} \cdot 4 \sigma_{\alpha \beta}^{2}\right]\right\}
\end{aligned}
$$

Note the surface gravity in the above results for Dp-brane is $\kappa_{p+2}^{2}$ which has the mass dimension $-p$. This is consistent with the mass dimension of the stress-energy tensor of Dp-brane i.e. $p+1$. Then one can read the general form of all the 2 nd order transport coefficients as

$$
\begin{aligned}
\eta \tau_{\pi} & =\frac{1}{2 \kappa_{p+2}^{2}}\left(\frac{1}{5-p}+\frac{1}{7-p} H_{\frac{5-p}{7-p}}\right) \frac{r_{H}^{2}}{L_{p}}, \\
\eta \tau_{\pi}^{*} & =\frac{1}{2 \kappa_{p+2}^{2}}\left(\frac{3(p-3)^{2}}{(5-p)(9-p)}-\frac{(p-3)^{2}}{(7-p)(9-p)} H_{\frac{5-p}{7-p}}\right) \frac{r_{H}^{2}}{L_{p}}, \\
\lambda_{1} & =\frac{1}{2 \kappa_{p+2}^{2}} \frac{1}{5-p} \frac{r_{H}^{2}}{L_{p}}, \quad \lambda_{2}=\frac{1}{2 \kappa_{p+2}^{2}}\left(-\frac{2}{5-p}+\frac{2}{7-p} H_{\frac{5-p}{7-p}}\right) \frac{r_{H}^{2}}{L_{p}}, \\
\zeta \tau_{\Pi} & =\frac{1}{2 \kappa_{p+2}^{2}}\left(\frac{2(p-3)^{2}}{p(5-p)(9-p)}+\frac{2(p-3)^{2}}{p(7-p)(9-p)} H_{\frac{5-p}{7-p}}\right) \frac{r_{H}^{2}}{L_{p}}, \\
\xi_{1} & =\frac{1}{2 \kappa_{p+2}^{2}} \frac{(p-3)^{2}}{p(5-p)(9-p)} \frac{r_{H}^{2}}{L_{p}}, \\
\xi_{2} & =\frac{1}{2 \kappa_{p+2}^{2}}\left(\frac{2(p-3)^{2}\left(3 p^{2}-17 p+18\right)}{p^{2}(5-p)(9-p)^{2}}+\frac{2(p-3)^{2}(5-p)}{p(7-p)(9-p)^{2}} H_{\frac{5-p}{7-p}}\right) \frac{r_{H}^{2}}{L_{p}} .
\end{aligned}
$$

Here $H_{\frac{5-p}{7-p}}$ is the Harmonic number which is defined as

$$
H_{\frac{a}{b}}=\frac{b}{a}+2 \sum_{n=1}^{\left[\frac{b-1}{2}\right]} \cos \left(\frac{2 \pi n a}{b}\right) \ln \sin \left(\frac{n \pi}{b}\right)-\frac{\pi}{2} \cot \left(\frac{\pi a}{b}\right)-\ln (2 b),
$$

where $a, b$ are positive integers with $a<b$. For the special cases of $1 \leq p \leq 4$, one has

$$
\begin{array}{ll}
H_{\frac{2}{3}}=\frac{3}{2}+\frac{\pi}{2 \sqrt{3}}-\frac{3}{2} \ln 3, & (p=1) \\
H_{\frac{3}{5}}=\frac{5}{3}+\frac{\pi}{2} \sqrt{1-\frac{2}{\sqrt{5}}}+\frac{5}{2 \sqrt{5}} \operatorname{arcoth} \sqrt{5}-\frac{5}{4} \ln 5, & (p=2)
\end{array}
$$




$$
\begin{array}{ll}
H_{\frac{1}{2}}=2-2 \ln 2, & (p=3) \\
H_{\frac{1}{3}}=3-\frac{\pi}{2 \sqrt{3}}-\frac{3}{2} \ln 3 . & (p=4)
\end{array}
$$

Please remember that $\eta \tau_{\pi}, \eta \tau_{\pi}^{*}, \lambda_{2}, \xi_{1}$ are not appropriate for $p=1$ and $\lambda_{1}$ is not suitable for both $p=1,2$ in eq. (6.12).

We find previous studies [49] and [47] also give general expressions on Dp-brane relaxation time. The author of [49] calculates the sound mode dispersion relation for Dp-branes and finds a relation between the shear and bulk relaxation time as in its equation (79). One can check that our results eq. (6.12) satisfy that relation. In equation (4.5) of [47], the author offers a general formula for the shear relaxation time of Dp-brane. If one compares the result of $\tau_{\pi}$ in eq. (6.12) with that from [47], one can see that the result in [47] misses a term of $\frac{1}{5-p}$. That is how $\tau_{\pi}$ of D2-brane in [47] disagrees with our result in eq. (6.10).

The way to express all the results for Dp-brane i.e. the energy density, pressure and the first order transport coefficients in eq. (4.3), as well as the second order transport coefficients listed in eq. (6.12) are all expressed via geometric parameters $2 \kappa_{p+2}^{2}, r_{H}$ and $L_{p}$. One can also reformulate the expressions in field theory language via 't Hooft coupling of the gauge theory on Dp-brane world-volume $\lambda_{p+1}$, the number of Dp-branes $N$ and the temperature of the boundary fluid $T . \lambda_{p+1}$ here is defined as $\lambda_{p+1}=g_{p+1}^{2} N \Lambda^{p-3}$, where $g_{p+1}^{2}=(2 \pi)^{p-2} g_{s} l_{s}^{p-3}$ is the gauge coupling of the effective field theory on Dp-brane worldvolume and $\Lambda=r / \alpha^{\prime}$ is the characteristic UV cutoff scale under the field theory limit. With the definitions of $\lambda_{p+1}, g_{p+1}^{2}, L_{p}$ eq. (2.5) and Hawking temperature eq. (4.4), we can translate the geometric language of expressing the results into field theory language. The results are listed in table 3.

\subsection{Relations between second order transport coefficients}

Now we would like to discuss the identities satisfied by the 2nd order transport coefficients calculated in this paper.

First is the Haack-Yarom (HY) relation $4 \lambda_{1}+\lambda_{2}=2 \eta \tau_{\pi}$. As it has been discussed in [13] that the HY relation has been verified to be valid for a wide range of cases except for the second order $\lambda_{G B}$ correction [28-30]. By the 2nd order stress-energy tensor of D1 eq. (6.7) and D2-brane eq. (6.9) we can see that the HY relation does not exist for these two cases. Because D1-brane does not support any tensor viscous term and D2-brane does not have the term $\sigma_{\langle\mu}{ }^{\rho} \sigma_{\nu\rangle \rho}$ which relates $\lambda_{1}$. Similar observation has also been made in [54] for U(1) charged conformal fluid. But the HY relation holds for D3 and D4-brane which can be seen from their 2nd order transport coefficients i.e. eqs. (6.3), (6.6). Thus we can conclude that the HY relation exists for Dp-brane with only $p>2$ and is also satisfied by those cases. If one does not consider D5 and D6-brane that do not have dual relativistic fluid, we find D4-brane as a new example that confirms the robustness of HY relation in nonconformal regime. 


\begin{tabular}{|c|c|}
\hline$\varepsilon$ & $\frac{9-p}{2}\left(\frac{4 \pi}{7-p}\right)^{2} \frac{2^{\frac{2(7-p)}{5-p}} \pi^{\frac{3-p}{5-p}}}{(7-p)^{\frac{11-p}{5-p}}} \Gamma\left(\frac{9-p}{2}\right)^{\frac{2}{5-p}} \lambda_{p+1}^{\frac{p-3}{5-p}} N^{2} \frac{T^{\frac{2(7-p)}{5-p}}}{\Lambda^{\frac{(p-3)^{2}}{5-p}}}$ \\
\hline $\mathfrak{p}$ & $\frac{5-p}{2}\left(\frac{4 \pi}{7-p}\right)^{2} \frac{2^{\frac{2(7-p)}{5-p}} \pi^{\frac{3-p}{5-p}}}{(7-p)^{\frac{11-p}{5-p}}} \Gamma\left(\frac{9-p}{2}\right)^{\frac{2}{5-p}} \lambda_{p+1}^{\frac{p-3}{5-p}} N^{2} \frac{T^{\frac{2(7-p)}{5-p}}}{\Lambda^{\frac{(p-3)^{2}}{5-p}}}$ \\
\hline$\eta$ & $\left(\frac{4 \pi}{7-p}\right) \frac{2^{\frac{2(7-p)}{5-p}} \pi^{\frac{3-p}{5-p}}}{(7-p)^{\frac{11-p}{5-p}}} \Gamma\left(\frac{9-p}{2}\right)^{\frac{2}{5-p}} \lambda_{p+1}^{\frac{p-3}{5-p}} N^{2} \frac{T^{\frac{9-p}{5-p}}}{\Lambda^{\frac{(p-3)^{2}}{5-p}}}$ \\
\hline$\zeta$ & $\frac{2(p-3)^{2}}{p(9-p)}\left(\frac{4 \pi}{7-p}\right) \frac{2^{\frac{2(7-p)}{5-p}} \pi^{\frac{3-p}{5-p}}}{(7-p)^{\frac{11-p}{5-p}}} \Gamma\left(\frac{9-p}{2}\right)^{\frac{2}{5-p}} \lambda_{p+1}^{\frac{p-3}{5-p}} N^{2} \frac{T^{\frac{9-p}{5-p}}}{\Lambda^{\frac{(p-p)^{2}}{5-p}}}$ \\
\hline$\eta \tau_{\pi}$ & $\left(\frac{1}{5-p}+\frac{1}{7-p} H_{\frac{5-p}{7-p}}\right) \frac{2^{\frac{2(7-p)}{5-p}} \pi^{\frac{3-p}{5-p}}}{(7-p)^{\frac{11-p}{5-p}}} \Gamma\left(\frac{9-p}{2}\right)^{\frac{2}{5-p}} \lambda_{p+1}^{\frac{p-3}{5-p}} N^{2} \frac{T^{\frac{4}{5-p}}}{\Lambda^{\frac{(p-3)^{2}}{5-p}}}$ \\
\hline$\eta \tau_{\pi}^{*}$ & $\left(\frac{3(p-3)^{2}}{(5-p)(9-p)}-\frac{(p-3)^{2}}{(7-p)(9-p)} H_{\frac{5-p}{7-p}}\right) \frac{2^{\frac{2(7-p)}{5-p}} \pi^{\frac{3-p}{5-p}}}{(7-p)^{\frac{11-p}{5-p}}} \Gamma\left(\frac{9-p}{2}\right)^{\frac{2}{5-p}} \lambda_{p+1}^{\frac{p-3}{5-p}} N^{2} \frac{T^{\frac{4}{5^{-p}}}}{\Lambda^{\frac{(p-3)^{2}}{5-p}}}$ \\
\hline$\lambda_{1}$ & $\frac{1}{5-p} \frac{2^{\frac{2(7-p)}{5-p}} \pi^{\frac{3-p}{5-p}}}{(7-p)^{\frac{11-p}{5-p}}} \Gamma\left(\frac{9-p}{2}\right)^{\frac{2}{5-p}} \lambda_{p+1}^{\frac{p-3}{5-p}} N^{2} \frac{T^{\frac{4}{5-p}}}{\Lambda^{\frac{(p-3)^{2}}{5-p}}}$ \\
\hline$\lambda_{2}$ & $\left(-\frac{2}{5-p}+\frac{2}{7-p} H_{\frac{5-p}{7-p}}\right) \frac{2^{\frac{2(7-p)}{5-p}} \pi^{\frac{3-p}{5-p}}}{(7-p)^{\frac{11-p}{5-p}}} \Gamma\left(\frac{9-p}{2}\right)^{\frac{2}{5-p}} \lambda_{p+1}^{\frac{p-3}{5-p}} N^{2} \frac{T^{\frac{4}{5-p}}}{\Lambda^{\frac{(p-3)}{5-p}}}$ \\
\hline$\zeta \tau_{\Pi}$ & $\left(\frac{2(p-3)^{2}}{p(5-p)(9-p)}+\frac{2(p-3)^{2}}{p(7-p)(9-p)} H_{\frac{5-p}{7-p}}\right) \frac{2^{\frac{2(7-p)}{5-p}} \pi^{\frac{3-p}{5-p}}}{(7-p)^{\frac{11-p}{5-p}}} \Gamma\left(\frac{9-p}{2}\right)^{\frac{2}{5-p}} \lambda_{p+1}^{\frac{p-3}{5-p}} N^{2} \frac{T^{\frac{4}{5-p}}}{\Lambda^{\frac{(p-3)^{2}}{5-p}}}$ \\
\hline$\xi_{1}$ & $\frac{(p-3)^{2}}{p(5-p)(9-p)} \frac{2^{\frac{2(7-p)}{5-p}} \pi^{\frac{3-p}{5-p}}}{(7-p)^{\frac{11-p}{5-p}}} \Gamma\left(\frac{9-p}{2}\right)^{\frac{2}{5-p}} \lambda_{p+1}^{\frac{p-3}{5-p}} N^{2} \frac{T^{\frac{4}{5-p}}}{\Lambda^{\frac{(p-3)^{2}}{5-p}}}$ \\
\hline$\xi_{2}$ & $\left(\frac{2(p-3)^{2}\left(3 p^{2}-17 p+18\right)}{p^{2}(5-p)(9-p)^{2}}+\frac{2(p-3)^{2}(5-p)}{p(7-p)(9-p)^{2}} H_{\frac{5-p}{7-p}}\right) \frac{2^{\frac{2(7-p)}{5-p}} \pi^{\frac{3-p}{5-p}}}{(7-p)^{\frac{11-p}{5-p}}} \Gamma\left(\frac{9-p}{2}\right)^{\frac{2}{5-p}} \lambda_{p+1}^{\frac{p-3}{5-p}} N^{2} \frac{T^{\frac{4}{5-p}}}{\Lambda^{\frac{(p-3)^{2}}{5-p}}}$ \\
\hline
\end{tabular}

Table 3. Reformulation of the results in field theory language. Remember that $\lambda_{3}$ and $\xi_{3}$ are both 0 .

The next are two relations that were first proposed by Romatschke in [55]:

$$
\begin{aligned}
\tau_{\pi} & =\tau_{\Pi}, \\
\xi_{1} & =\frac{1-3 c_{s}^{2}}{3} \lambda_{1} .
\end{aligned}
$$

It has been shown that the above are satisfied by the 2 nd order transport coefficients of compactified D4-brane in [13]. What we want to point out first is eq. (6.18) is not suitable for D1-brane and eq. (6.19) is only valid for $p>2$. Then if we check the above two relations using eq. (6.12), we can find eq. (6.18) still holds while eq. (6.19) is not. This is because Romatschke only considered the hydrodynamics of $4 \mathrm{~d}$ in [55]. The second relation has to be generalized to arbitrary dimensions as

$$
\xi_{1}=\frac{1}{d-1}\left(1-(d-1) c_{s}^{2}\right) \lambda_{1}, \quad(d>3)
$$

where $d=p+1$ is the dimension of the relativistic fluid. As one can check that eq. (6.12) satisfies eq. (6.20). 
Besides the two relations discussed in the last paragraph, [55] also proposed many other relations between the 2 nd order coefficients, e.g.

$$
\tau_{\pi}^{*}=-\left(1-3 c_{s}^{2}\right) \tau_{\pi}, \quad \xi_{2}=\frac{1-3 c_{s}^{2}}{3} 2 c_{s}^{2} \eta \tau_{\pi} .
$$

But it has been pointed out in [35] by Kleinert and Probst that these two relations may miss the term of $\lambda_{1}$, and the correct form should be

$$
\eta \tau_{\pi}^{*}=\left(1-3 c_{s}^{2}\right)\left(4 \lambda_{1}-\eta \tau_{\pi}\right), \quad \xi_{2}=\frac{2}{9}\left(1-3 c_{s}^{2}\right)\left[3 c_{s}^{2} \eta \tau_{\pi}+\left(1-6 c_{s}^{2}\right) 2 \lambda_{1}\right] .
$$

Kleinert and Probst have also checked that the above two relations are satisfied by the compactified D4-brane $[13,45,51]$. But if we are in dimensions other than 4 like the case in this paper, the above Kleinert-Probst (KP) relations eq. (6.22) should be generalized to

$$
\begin{aligned}
\eta \tau_{\pi}^{*} & =\left(1-(d-1) c_{s}^{2}\right)\left(4 \lambda_{1}-\eta \tau_{\pi}\right), & & (d>3) \\
\xi_{2} & =\frac{2}{(d-1)^{2}}\left(1-(d-1) c_{s}^{2}\right)\left[\left(1-2(d-1) c_{s}^{2}\right) 2 \lambda_{1}+(d-1) c_{s}^{2} \eta \tau_{\pi}\right] . & & (d>3)
\end{aligned}
$$

As one can check, the above two generalized KP relations are indeed satisfied by the 2nd order transport coefficients of Dp-brane eq. (6.12) in this paper. Since the above two relations also have $\lambda_{1}$, so they are only appropriate for the cases with $p>2$ (or $d>3$ ), like the HY relation and the generalized Romatschke relation eq. (6.20).

There are some other nontrivial relations among the 2nd order thermodynamical transport coefficients ${ }^{5}$ proposed in [55], which are

$$
\begin{aligned}
\kappa^{*} & =-\frac{1-3 c_{s}^{2}}{2 c_{s}^{2}} \kappa, & \xi_{3} & =\frac{1-3 c_{s}^{2}}{3} \lambda_{3}, \\
\xi_{5} & =\frac{1-3 c_{s}^{2}}{3} \kappa, & \xi_{6} & =\frac{1-3 c_{s}^{2}}{3 c_{s}^{2}} \kappa .
\end{aligned}
$$

Kleinert and Probst check the above relations to be true for the $5 \mathrm{~d}$ Chamblin-Reall background using the method of [37]. According to the generalization from eq. (6.22) to eq. (6.23), we think the above relations may also need to be generalized in arbitrary dimensions to:

$$
\begin{array}{rlrl}
\kappa^{*}=-\frac{1-(d-1) c_{s}^{2}}{2 c_{s}^{2}} \kappa, & \xi_{3} & =\frac{1-(d-1) c_{s}^{2}}{d-1} \lambda_{3}, \\
\xi_{5}=\frac{1-(d-1) c_{s}^{2}}{d-1} \kappa, & \xi_{6}=\frac{1-(d-1) c_{s}^{2}}{(d-1) c_{s}^{2}} \kappa .
\end{array}
$$

Although we do not know for now whether eq. (6.25) is completely correct since it needs to be checked by strict calculation in nonconformal backgrounds. We hope future studies will give us a satisfied answer.

\footnotetext{
${ }^{5}$ As it has been classified in [52] that there are 8 thermodynamical transport coefficients out of the 15 second order coefficients which do not cause the increase of entropy.
} 


\subsection{Test of Kanitscheider-Skenderis proposal}

Kanitscheider and Skenderis proposed a way of generating the second order part of nonconformal stress-energy tensor from that of a higher dimensional conformal relativistic fluid [37]. With the help of the results in this paper, we can test whether their method is correct.

First we need the result of stress-energy tensor of conformal relativistic fluid in various dimensions. The following stress-energy tensor summarizes all the results from $[2-7,10$, $11,15-18]$ and offers the stress-energy tensor of $d$-dimensional conformal relativistic fluid that corresponds to $\mathrm{AdS}_{d+1}$ black hole:

$$
\begin{aligned}
T_{\mu \nu}=\frac{1}{2 \kappa_{d+1}^{2}}\left\{\frac{r_{H}^{d}}{L^{d+1}}\left[(d-1) u_{\mu} u_{\nu}+P_{\mu \nu}\right]-\left(\frac{r_{H}}{L}\right)^{d-1} 2 \sigma_{\mu \nu}\right. \\
+\frac{r_{H}^{d-2}}{L^{d-3}}\left[( \frac { 1 } { 2 } + \frac { 1 } { d } H _ { \frac { 2 } { d } } ) 2 \left(\left\langle D \sigma_{\mu \nu\rangle}+\frac{1}{d-1} \sigma_{\mu \nu} \partial u\right)+\frac{1}{2} \cdot 4 \sigma_{\langle\mu}{ }^{\rho} \sigma_{\nu\rangle \rho}\right.\right. \\
\left.\left.\quad+\left(\frac{2}{d} H_{\frac{2}{d}}-1\right) 2 \sigma_{\langle\mu}{ }^{\rho} \Omega_{\nu\rangle \rho}+\frac{2}{d-2}\left(R_{\langle\mu \nu\rangle}-2 u^{\rho} u^{\sigma} R_{\rho\langle\mu \nu\rangle \sigma}\right)\right]\right\},
\end{aligned}
$$

where $\frac{1}{2 \kappa_{d+1}^{2}}, L$ and $r_{H}$ are separately the surface gravity constant, the curvature scale and the location of horizon of $\mathrm{AdS}_{d+1}$ black hole background. The energy density, pressure and the transport coefficients can then be read $\operatorname{as}^{6}$

$$
\begin{aligned}
& \varepsilon=\frac{1}{2 \kappa_{d+1}^{2}}(d-1) \frac{r_{H}^{d}}{L^{d+1}}, \quad \quad \mathfrak{p}=\frac{1}{2 \kappa_{d+1}^{2}} \frac{r_{H}^{d}}{L^{d+1}}, \\
& \eta \tau_{\pi}=\frac{1}{2 \kappa_{d+1}^{2}}\left(\frac{1}{2}+\frac{1}{d} H_{\frac{2}{d}}\right) \frac{r_{H}^{d-2}}{L^{d-3}}, \quad \kappa=\frac{1}{2 \kappa_{d+1}^{2}} \frac{2}{d-2} \frac{r_{H}^{d-2}}{L^{d-3}}, \\
& \lambda_{1}=\frac{1}{2 \kappa_{d+1}^{2}} \frac{1}{2} \frac{r_{H}^{d-2}}{L^{d-3}}, \quad \lambda_{2}=\frac{1}{2 \kappa_{d+1}^{2}}\left(\frac{2}{d} H_{\frac{2}{d}}-1\right) \frac{r_{H}^{d-2}}{L^{d-3}}, \quad \lambda_{3}=0 . \\
& \eta=\frac{1}{2 \kappa_{d+1}^{2}} \frac{r_{H}^{d-1}}{L^{d-1}}
\end{aligned}
$$

The Hawking temperature, entropy density and shear to entropy ratio are

$$
T=\frac{d}{4 \pi} \frac{r_{H}}{L^{2}}, \quad s=\frac{1}{2 \kappa_{d+1}^{2}} 4 \pi \frac{r_{H}^{d-1}}{L^{d-1}}, \quad \frac{\eta}{s}=\frac{1}{4 \pi} .
$$

Following the method that proposed by Kanitscheider and Skenderis in [37], the 2nd order viscous terms in eq. (6.26) will transform like

$$
\begin{aligned}
\left\langle D \sigma_{\mu \nu\rangle}\right. & \rightarrow\left\langle D \sigma_{\mu \nu\rangle}+\chi P_{\mu \nu} D(\partial u),\right. & \sigma_{\mu \nu} \partial u & \rightarrow \sigma_{\mu \nu} \partial u+\chi P_{\mu \nu}(\partial u)^{2}, \\
\sigma_{\langle\mu}{ }^{\rho} \sigma_{\nu\rangle \rho} & \rightarrow \sigma_{\langle\mu}{ }^{\rho} \sigma_{\nu\rangle \rho}+\chi P_{\mu \nu} \sigma_{\alpha \beta}^{2}, & \sigma_{\langle\mu}{ }^{\rho} \Omega_{\nu\rangle \rho} & \rightarrow \sigma_{\langle\mu}{ }^{\rho} \Omega_{\nu\rangle \rho} .
\end{aligned}
$$

Here $\chi$ is defined as

$$
\chi=\frac{\tilde{d}-d}{(\tilde{d}-1)(d-1)} .
$$

\footnotetext{
${ }^{6}$ The result in [11] is expressed via $H_{\frac{2}{d}-1}$, which can be related to $H_{\frac{2}{d}}$ by the recurrence relation of Harmonic number: $H_{\alpha}=H_{\alpha-1}+\frac{1}{\alpha}$.
} 
We denote $\tilde{d}$ as the dimension of conformal fluid that is dual to $\operatorname{AdS}_{\tilde{d}+1}$ black hole background, and $d=p+1$ as the dimension of the nonconformal fluid which correspond to Dp-brane, with $\tilde{d}>d$. Now we want to see with the transformation rule eq. (6.29), whether we can get the 2 nd order part of stress-energy tensor for nonconformal $d$-dimensional fluid from that of a $\tilde{d}$-dimensional conformal fluid. The 2 nd order part stress-energy tensor of $\tilde{d}$-dimensional conformal fluid is

$$
\widetilde{\eta \tau_{\pi}} \cdot 2\left(\left\langle D \sigma_{\mu \nu\rangle}+\frac{1}{\tilde{d}-1} \sigma_{\mu \nu} \partial u\right)+\tilde{\lambda}_{1} \cdot 4 \sigma_{\langle\mu}{ }^{\rho} \sigma_{\nu\rangle \rho}+\tilde{\lambda}_{2} \cdot 2 \sigma_{\langle\mu}{ }^{\rho} \Omega_{\nu\rangle \rho},\right.
$$

where we also write the 2 nd order coefficients of $\mathrm{AdS}_{\tilde{d}+1}$ black hole background with a tilde. Also note that here we do not keep quantities with dimension like $r_{H}$ and $L$, since the Kanitscheider-Skenderis proposal only involves with numerical value of the 2 nd order transport coefficients. After using the transformation rule eq. (6.29), the above will become

$$
\begin{aligned}
& \widetilde{\eta \tau_{\pi}} \cdot 2\left(\left\langle D \sigma_{\mu \nu\rangle}+\frac{1}{d-1} \sigma_{\mu \nu} \partial u\right)-(d-1) \chi \widetilde{\eta \tau_{\pi}} \frac{2 \sigma_{\mu \nu} \partial u}{d-1}+\tilde{\lambda}_{1} \cdot 4 \sigma_{\langle\mu}{ }^{\rho} \sigma_{\nu\rangle \rho}\right. \\
& +\tilde{\lambda}_{2} \cdot 2 \sigma_{\langle\mu}{ }^{\rho} \Omega_{\nu\rangle \rho}+P_{\mu \nu}\left(2 \chi \widetilde{\eta \tau_{\pi}} D(\partial u)+\frac{2 \chi \widetilde{\eta \tau_{\pi}}}{\tilde{d}-1}(\partial u)^{2}+\chi \tilde{\lambda}_{1} \cdot 4 \sigma_{\alpha \beta}^{2}\right) .
\end{aligned}
$$

Thus we get the relations between the 2nd order transport coefficients of nonconformal fluid and that of the conformal fluid:

$$
\begin{aligned}
& \eta \tau_{\pi}=\widetilde{\eta \tau_{\pi}}, \quad \eta \tau_{\pi}^{*}=-(d-1) \chi \widetilde{\eta \tau_{\pi}}, \quad \lambda_{1}=\tilde{\lambda}_{1}, \quad \lambda_{2}=\tilde{\lambda}_{2}, \\
& \zeta \tau_{\Pi}=2 \chi \widetilde{\eta \tau_{\pi}}, \quad \xi_{1}=\chi \tilde{\lambda}_{1}, \quad \xi_{2}=\frac{2 \chi}{\tilde{d}-1} \widetilde{\eta \tau_{\pi}} .
\end{aligned}
$$

Now we will use the results of D1, D2 and D4-brane to test whether the relations eq. (6.33) are reliable. Since the D4-brane result can be got from $\mathrm{AdS}_{7}$ black hole background, so we have $\tilde{d}=6, d=p+1=5$ and $\chi=1 / 20$. The 2 nd order transport coefficients for $\mathrm{AdS}_{7}$ black hole are

$$
\widetilde{\eta \tau_{\pi}}=\frac{1}{2}+\frac{1}{6} H_{\frac{1}{3}}, \quad \tilde{\lambda}_{1}=\frac{1}{2}, \quad \tilde{\lambda}_{2}=-1+\frac{1}{3} H_{\frac{1}{3}} .
$$

Thus we can get from eq. (6.33) that

$$
\begin{array}{rlrlrl}
\eta \tau_{\pi} & =\frac{1}{2}+\frac{1}{6} H_{\frac{1}{3}}, & \eta \tau_{\pi}^{*}=-\frac{1}{10}-\frac{1}{30} H_{\frac{1}{3}}, & \lambda_{1}=\frac{1}{2}, & \lambda_{2}=-1+\frac{1}{3} H_{\frac{1}{3}}, \\
\zeta \tau_{\Pi}=\frac{1}{20}+\frac{1}{60} H_{\frac{1}{3}}, & \xi_{1}=\frac{1}{40}, & \xi_{2}=\frac{1}{100}+\frac{1}{300} H_{\frac{1}{3}} . &
\end{array}
$$

Since a global scaling factor for the above results do not matter, we can multiply a scaling factor 2 to the above results and get

$$
\begin{array}{rlrlrl}
\eta \tau_{\pi} & =1+\frac{1}{3} H_{\frac{1}{3}}, & \eta \tau_{\pi}^{*}=-\frac{1}{5}-\frac{1}{15} H_{\frac{1}{3}}, & \lambda_{1} & =1, & \lambda_{2}=-2+\frac{2}{3} H_{\frac{1}{3}}, \\
\zeta \tau_{\Pi}=\frac{1}{10}+\frac{1}{30} H_{\frac{1}{3}}, & \xi_{1}=\frac{1}{20}, & \xi_{2}=\frac{1}{50}+\frac{1}{150} H_{\frac{1}{3}} . &
\end{array}
$$


Compare the above with eq. (6.12) for the case $p=4$, we can see that $\eta \tau_{\pi}, \zeta \tau_{\Pi}, \lambda_{1,2}, \xi_{1}$ are the same as the results of D4-brane, but $\eta \tau_{\pi}^{*}$ and $\xi_{2}$ do not match.

By the same token, one can check the situations of D1 and D2-brane. The reduced bulk gravity of D1-brane relates to $\mathrm{AdS}_{4}$ black hole background, after using the relations eq. (6.33) and multiplying a scaling factor 2 one can reproduce exactly the second order transport coefficients for D1-brane. The second order transport coefficients of D2-brane should be got from that of $\mathrm{AdS}_{6}$ black hole by using eq. (6.33). ${ }^{7}$ But one will see that the results got this way can not match any transport coefficient of D2-brane in eq. (6.12).

The authors of [35] derive out the exact analytical results of the second order transport coefficients for $5 \mathrm{~d}$ Chamblin-Reall background and use the leading nonconformal corrections of the results to compare with [36]. It is interesting to note that they also find $\eta \tau_{\pi}^{*}$ and $\xi_{2}$ in [36] are wrong. The reason they think is that [36] uses eq. (6.21) (first proposed in [55]) to get $\eta \tau_{\pi}^{*}$ and $\xi_{2}$ whose correct form should be eq. (6.22). Considering that [55] derived the relations eq. (6.21) also using the method of [37], we may draw the conclusion that the method of getting the 2nd order transport coefficients of nonconformal Dp-brane from AdS black hole background as suggested in [37] is applicable only for some special cases. It is better to use the standard methods like the Minkowskian AdS/CFT prescription or the fluid/gravity correspondence to calculate the 2nd order transport coefficients for nonconformal backgrounds.

\subsection{Dispersion relations}

At last, we would like to offer the dispersion relations for the dual relativistic fluid of Dpbrane with $1 \leq p \leq 4$. One needs to expand $r_{H}(x)$ and $\beta_{i}(x)$ as $r_{H}(x)=r_{H}(0)+\delta r_{H} \mathrm{e}^{i k_{\mu} x^{\mu}}$ and $\beta_{i}(x)=\delta \beta_{i} \mathrm{e}^{i k_{\mu} x^{\mu}}$, respectively. Then put them into the conservation equation of the stress-energy tensor of Dp-brane $\partial^{\mu} T_{\mu \nu}=0$ and find the coefficient matrix of the vector $\left(\delta r_{H}, \delta \beta_{i}\right)^{T}$ where the subscript "T" stands for taking the transpose. Then the determinant of that coefficient matrix equate to 0 will give two equations from which we can solve the transverse and the longitudinal modes of the dispersion relations (denoted separately as $\omega_{T}$ and $\left.\omega_{L}\right)$. The result is

$$
\begin{aligned}
\omega_{T}= & -i \frac{1}{7-p} \frac{L_{p}^{\frac{7-p}{2}}}{r_{H}^{\frac{5-p}{2}}} k^{2}-i \frac{(7-p)+(5-p) H_{\frac{5-p}{7-p}}}{(5-p)(7-p)^{3}} \frac{L_{p}^{\frac{3(7-p)}{2}}}{r_{H}^{\frac{3(5-p)}{2}}} k^{4}, \quad(p \neq 1) \\
\omega_{L}= & \pm \sqrt{\frac{5-p}{9-p}} k-i \frac{4}{(7-p)(9-p)} \frac{L_{p}^{\frac{7-p}{2}}}{r_{H}^{\frac{5-p}{2}}} k^{2} \pm \frac{4(5-p)^{\frac{1}{2}}\left(1+H_{\frac{5-p}{7-p}}\right)}{(7-p)^{2}(9-p)^{\frac{3}{2}}} \frac{L_{p}^{7-p}}{r_{H}^{5-p}} k^{3} \\
& -i \frac{32\left(7-p+(5-p) H_{\frac{5-p}{7-p}}\right)}{(5-p)(7-p)^{3}(9-p)^{2}} \frac{L_{p}^{\frac{3(7-p)}{2}}}{r_{H}^{\frac{3(5-p)}{2}}} k^{4} .
\end{aligned}
$$

Here $k=|\vec{k}|$ and also note that $p$ can not be 1 for the transverse mode.

\footnotetext{
${ }^{7}$ One will need the reflection relation of Harmonic number $H_{1-\alpha}=H_{\alpha}+\pi \cot \pi \alpha+\frac{1}{1-\alpha}-\frac{1}{\alpha}$ in comparing the results got from eq. (6.33) with the second order transport coefficients of D2-brane in eq. (6.12).
} 


\section{Summary and outlook}

In this paper, we have derived all the dynamical second order transport coefficients for Dp-brane using fluid/gravity correspondence. D5 and D6-brane do not have physical dual relativistic fluid; D3-brane is dual to $4 \mathrm{~d}$ conformal fluid of which the results are already well known in the literature; thus the new results are mainly for D1, D2 and D4-brane, which correspond to nonconformal fluid of dimension 2, 3 and 5.

The validity of the identities among 2nd order transport coefficients has exceptions: $\tau_{\pi}=\tau_{\Pi}$ is not valid for D1-brane; the HY relation, the generalized Romatschke eq. (6.20) and KP eq. (6.23) relations are appropriate for Dp-brane with $p>2$, or for relativistic fluid of dimension $d>3$. The general form of the 2nd order transport coefficients of Dpbrane eq. (6.12) satisfies the HY, Romatschke and KP relations. We also think that the constraint relations between the 2 nd order transport coefficients, which were first proposed in [56] and rewritten in a more useful form in [52], need to be generalized if the relativistic fluid is not in $4 \mathrm{~d}$. So one should be careful when using them in dimensions other than 4 .

This work leaves us some interesting problems to explore. First, we can continue to study the thermodynamical 2nd order transport coefficients for Dp-brane via the real-time AdS/CFT correspondence. Second, D5 and D6-brane are not dual to any relativistic fluid. So we may need to use the NS5-brane if we want to study the relativistic fluid of dimension larger than 5. NS5-brane, if it has, will be dual to a nonconformal relativistic fluid of 6d. Finally, we can tell from the results of D4-brane and compactified D4-brane that the compactification on the brane will change the spatial dimension of the fluid thus the 2nd order transport coefficients relating viscous scalars, i.e., $\eta \tau_{\pi}^{*}, \zeta \tau_{\Pi}$ and $\xi_{1,2}$. So we can compactify more directions on D4-brane to get nonconformal fluids of dimension 2 and 3 , and one can also compactify the other Dp-branes. It will be interesting to see whether D3-brane will correspond to a nonconformal fluid after compactification.

Through $[13,51]$ and this work, we have successfully generalized the original discussion of fluid/gravity correspondence to nonconformal regime for various dimensions. By the stress-energy tensor of general dimension eq. (6.11), we actually offers a group of nonconformal relativistic fluid models. We hope these models can be used to get the analytic solutions by using the framework of [57-63] thus will give us more hints on the phenomena of relativistic heavy ion collider.

\section{Acknowledgments}

First, we would like to thank $\mathrm{Yu} \mathrm{Lu}$ for his generous help on Mathematica code and programming. We also want to thank Zoltan Bajnok, Haryanto Siahaan for very helpful discussions, Ze-Fang Jiang for introducing some references on exact solutions in relativistic hydrodynamics, and Michael Abbott for his kind help on improving presentation. This work is supported by the Hungarian National Science Fund NKFIH (under K116505), a Lendület Grant and the Young Scientists Fund of the National Natural Science Foundation of China (Grant No. 11805002). 


\section{A Dimensional reduction of bulk metric}

We will do the dimensional reduction on the following 10d metric

$$
d s^{2}=e^{2 \alpha_{1} A} g_{M N} d x^{M} d x^{N}+L_{p}^{2} e^{2 \alpha_{2} A} d \Omega_{8-p}^{2},
$$

where the dimension of the $\left\{x^{M}\right\}$ part is $(p+2)$-dimensional, $A=A\left(x^{M}\right)$ only depend on coordinates of the first $p+2$ dimensions, the second part is a $(8-p)$-dimensional unit sphere with the metric $\gamma_{a b} . \alpha_{1,2}, L_{p}$ are all constants. The components of connection are

$$
\begin{aligned}
\widehat{\Gamma}_{N P}^{M} & =\Gamma_{N P}^{M}+\alpha_{1}\left(\delta_{N}^{M} \partial_{P} A+\delta_{P}^{M} \partial_{N} A-g_{N P} \nabla^{M} A\right), \\
\widehat{\Gamma}_{a b}^{M} & =-\alpha_{2} L_{p}^{2} e^{\left(-2 \alpha_{1}+2 \alpha_{2}\right) A} \nabla^{M} A \gamma_{a b}, \\
\widehat{\Gamma}_{M b}^{a} & =\alpha_{2} \partial_{M} A \delta_{b}^{a}, \\
\widehat{\Gamma}_{b c}^{a} & =\Gamma_{b c}^{a} .
\end{aligned}
$$

The Ricci tensor is

$$
\begin{array}{r}
\widehat{R}_{M N}=R_{M N}-\left[p \alpha_{1}+(8-p) \alpha_{2}\right] \nabla_{M} \nabla_{N} A-\alpha_{1} g_{M N} \nabla^{2} A+\left[p \alpha_{1}^{2}+2(8-p) \alpha_{1} \alpha_{2}\right. \\
\left.-(8-p) \alpha_{2}^{2}\right] \nabla_{M} A \nabla_{N} A-\left[p \alpha_{1}^{2}+(8-p) \alpha_{1} \alpha_{2}\right] g_{M N}(\nabla A)^{2}, \\
\widehat{R}_{a b}=(7-p) \gamma_{a b}-L_{p}^{2} e^{\left(-2 \alpha_{1}+2 \alpha_{2}\right) A}\left[\alpha_{2} \nabla^{2} A+\left(p \alpha_{1} \alpha_{2}+(8-p) \alpha_{2}^{2}\right)(\nabla A)^{2}\right] \gamma_{a b} .
\end{array}
$$

The Ricci scalar is

$$
\begin{array}{r}
\widehat{R}=\frac{(7-p)(8-p)}{L_{p}^{2}} e^{-2 \alpha_{2} A}+e^{-2 \alpha_{1} A}\left[R-\left((2 p+2) \alpha_{1}+(16-2 p) \alpha_{2}\right) \nabla^{2} A\right. \\
\left.-\left(\left(p^{2}+p\right) \alpha_{1}^{2}+\left(16 p-2 p^{2}\right) \alpha_{1} \alpha_{2}+(8-p)(9-p) \alpha_{2}^{2}\right)(\nabla A)^{2}\right]
\end{array}
$$

The quantities with a hat like $\widehat{\Gamma}$ and $\widehat{R}$ are 10-dimensional quantities while those without like $R$ are $(p+2)$-dimensional ones.

Open Access. This article is distributed under the terms of the Creative Commons Attribution License (CC-BY 4.0), which permits any use, distribution and reproduction in any medium, provided the original author(s) and source are credited.

\section{References}

[1] D.T. Son and A.O. Starinets, Minkowski space correlators in AdS/CFT correspondence: recipe and applications, JHEP 09 (2002) 042 [hep-th/0205051] [INSPIRE].

[2] G. Policastro, D.T. Son and A.O. Starinets, From AdS/CFT correspondence to hydrodynamics, JHEP 09 (2002) 043 [hep-th/0205052] [INSPIRE].

[3] G. Policastro, D.T. Son and A.O. Starinets, From AdS/CFT correspondence to hydrodynamics. 2. Sound waves, JHEP 12 (2002) 054 [hep-th/0210220] [InSPIRE].

[4] R. Baier, P. Romatschke, D.T. Son, A.O. Starinets and M.A. Stephanov, Relativistic viscous hydrodynamics, conformal invariance and holography, JHEP 04 (2008) 100 [arXiv:0712.2451] [INSPIRE]. 
[5] E. Barnes, D. Vaman, C. Wu and P. Arnold, Real-time finite-temperature correlators from AdS/CFT, Phys. Rev. D 82 (2010) 025019 [arXiv:1004.1179] [INSPIRE].

[6] P. Arnold, D. Vaman, C. Wu and W. Xiao, Second order hydrodynamic coefficients from 3-point stress tensor correlators via AdS/CFT, JHEP 10 (2011) 033 [arXiv:1105.4645] [INSPIRE].

[7] S. Bhattacharyya, V.E. Hubeny, S. Minwalla and M. Rangamani, Nonlinear fluid dynamics from gravity, JHEP 02 (2008) 045 [arXiv: 0712.2456] [INSPIRE].

[8] S. Bhattacharyya et al., Local fluid dynamical entropy from gravity, JHEP 06 (2008) 055 [arXiv:0803.2526] [INSPIRE].

[9] S. Bhattacharyya, R. Loganayagam, S. Minwalla, S. Nampuri, S.P. Trivedi and S.R. Wadia, Forced fluid dynamics from gravity, JHEP 02 (2009) 018 [arXiv: 0806.0006] [INSPIRE].

[10] M. Haack and A. Yarom, Nonlinear viscous hydrodynamics in various dimensions using AdS/CFT, JHEP 10 (2008) 063 [arXiv:0806.4602] [INSPIRE].

[11] S. Bhattacharyya, R. Loganayagam, I. Mandal, S. Minwalla and A. Sharma, Conformal nonlinear fluid dynamics from gravity in arbitrary dimensions, JHEP 12 (2008) 116 [arXiv: 0809.4272] [INSPIRE].

[12] J. Erdmenger, M. Haack, M. Kaminski and A. Yarom, Fluid dynamics of R-charged black holes, JHEP 01 (2009) 055 [arXiv: 0809.2488] [INSPIRE].

[13] C. Wu, Y. Chen and M. Huang, Fluid/gravity correspondence: second order transport coefficients in compactified D4-branes, JHEP 01 (2017) 118 [arXiv:1604.07765] [INSPIRE].

[14] J.I. Kapusta and T. Springer, Shear transport coefficients from gauge/gravity correspondence, Phys. Rev. D 78 (2008) 066017 [arXiv:0806.4175] [INSPIRE].

[15] C.P. Herzog, The hydrodynamics of M-theory, JHEP 12 (2002) 026 [hep-th/0210126] [INSPIRE].

[16] M. Natsuume and T. Okamura, Causal hydrodynamics of gauge theory plasmas from AdS/CFT duality, Phys. Rev. D 77 (2008) 066014 [Erratum ibid. D 78 (2008) 089902] [arXiv: 0712.2916] [INSPIRE].

[17] M. Natsuume and T. Okamura, A note on causal hydrodynamics for M-theory branes, Prog. Theor. Phys. 120 (2008) 1217 [arXiv:0801.1797] [INSPIRE].

[18] M. Van Raamsdonk, Black hole dynamics from atmospheric science, JHEP 05 (2008) 106 [arXiv: 0802.3224] [INSPIRE].

[19] A. Buchel, J.T. Liu and A.O. Starinets, Coupling constant dependence of the shear viscosity in $N=4$ supersymmetric Yang-Mills theory, Nucl. Phys. B 707 (2005) 56 [hep-th/0406264] [INSPIRE].

[20] P. Benincasa and A. Buchel, Transport properties of $N=4$ supersymmetric Yang-Mills theory at finite coupling, JHEP 01 (2006) 103 [hep-th/0510041] [INSPIRE].

[21] A. Buchel and M. Paulos, Relaxation time of a CFT plasma at finite coupling, Nucl. Phys. B 805 (2008) 59 [arXiv:0806.0788] [INSPIRE].

[22] A. Buchel and M. Paulos, Second order hydrodynamics of a CFT plasma from boost invariant expansion, Nucl. Phys. B $\mathbf{8 1 0}$ (2009) 40 [arXiv:0808.1601] [InSPIRE].

[23] A. Buchel, R.C. Myers, M.F. Paulos and A. Sinha, Universal holographic hydrodynamics at finite coupling, Phys. Lett. B 669 (2008) 364 [arXiv:0808.1837] [INSPIRE].

[24] M. Brigante, H. Liu, R.C. Myers, S. Shenker and S. Yaida, Viscosity bound violation in higher derivative gravity, Phys. Rev. D 77 (2008) 126006 [arXiv:0712.0805] [INSPIRE]. 
[25] M. Brigante, H. Liu, R.C. Myers, S. Shenker and S. Yaida, The viscosity bound and causality violation, Phys. Rev. Lett. 100 (2008) 191601 [arXiv:0802.3318] [INSPIRE].

[26] E. Shaverin and A. Yarom, Universality of second order transport in Gauss-Bonnet gravity, JHEP 04 (2013) 013 [arXiv: 1211.1979] [INSPIRE].

[27] S. Grozdanov and A.O. Starinets, On the universal identity in second order hydrodynamics, JHEP 03 (2015) 007 [arXiv: 1412.5685] [INSPIRE].

[28] E. Shaverin, A breakdown of a universal hydrodynamic relation in Gauss-Bonnet gravity, arXiv: 1509.05418 [INSPIRE].

[29] S. Grozdanov and A.O. Starinets, Zero-viscosity limit in a holographic Gauss-Bonnet liquid, Theor. Math. Phys. 182 (2015) 61 [Teor. Mat. Fiz. 182 (2014) 76] [inSPIRE].

[30] S. Grozdanov and A.O. Starinets, Second-order transport, quasinormal modes and zero-viscosity limit in the Gauss-Bonnet holographic fluid, JHEP 03 (2017) 166 [arXiv: 1611.07053] [INSPIRE].

[31] S.S. Gubser, A. Nellore, S.S. Pufu and F.D. Rocha, Thermodynamics and bulk viscosity of approximate black hole duals to finite temperature quantum chromodynamics, Phys. Rev. Lett. 101 (2008) 131601 [arXiv:0804.1950] [INSPIRE].

[32] S.S. Gubser, S.S. Pufu and F.D. Rocha, Bulk viscosity of strongly coupled plasmas with holographic duals, JHEP 08 (2008) 085 [arXiv: 0806.0407] [INSPIRE].

[33] D. Li, S. He and M. Huang, Temperature dependent transport coefficients in a dynamical holographic QCD model, JHEP 06 (2015) 046 [arXiv: 1411.5332] [INSPIRE].

[34] S.I. Finazzo, R. Rougemont, H. Marrochio and J. Noronha, Hydrodynamic transport coefficients for the non-conformal quark-gluon plasma from holography, JHEP 02 (2015) 051 [arXiv: 1412.2968] [INSPIRE].

[35] P. Kleinert and J. Probst, Second-order hydrodynamics and universality in non-conformal holographic fluids, JHEP 12 (2016) 091 [arXiv:1610.01081] [INSPIRE].

[36] F. Bigazzi and A.L. Cotrone, An elementary stringy estimate of transport coefficients of large temperature QCD, JHEP 08 (2010) 128 [arXiv: 1006.4634] [INSPIRE].

[37] I. Kanitscheider and K. Skenderis, Universal hydrodynamics of non-conformal branes, JHEP 04 (2009) 062 [arXiv:0901.1487] [InSPIRE].

[38] P. Romatschke, New developments in relativistic viscous hydrodynamics, Int. J. Mod. Phys. E 19 (2010) 1 [arXiv:0902.3663] [InSPIRE].

[39] A. Buchel, $N=2 *$ hydrodynamics, Nucl. Phys. B 708 (2005) 451 [hep-th/0406200] [INSPIRE].

[40] P. Benincasa, A. Buchel and A.O. Starinets, Sound waves in strongly coupled non-conformal gauge theory plasma, Nucl. Phys. B 733 (2006) 160 [hep-th/0507026] [INSPIRE].

[41] A. Buchel and C. Pagnutti, Bulk viscosity of $N=2 *$ plasma, Nucl. Phys. B 816 (2009) 62 [arXiv: 0812.3623] [INSPIRE].

[42] A. Buchel, Transport properties of cascading gauge theories, Phys. Rev. D 72 (2005) 106002 [hep-th/0509083] [INSPIRE].

[43] A. Buchel, Bulk viscosity of gauge theory plasma at strong coupling, Phys. Lett. B 663 (2008) 286 [arXiv: 0708.3459] [INSPIRE].

[44] A. Parnachev and A. Starinets, The silence of the little strings, JHEP 10 (2005) 027 [hep-th/0506144] [INSPIRE]. 
[45] P. Benincasa and A. Buchel, Hydrodynamics of Sakai-Sugimoto model in the quenched approximation, Phys. Lett. B 640 (2006) 108 [hep-th/0605076] [INSPIRE].

[46] J. Mas and J. Tarrio, Hydrodynamics from the Dp-brane, JHEP 05 (2007) 036 [hep-th/0703093] [INSPIRE].

[47] M. Natsuume, Causal hydrodynamics and the membrane paradigm, Phys. Rev. D 78 (2008) 066010 [arXiv:0807.1392] [INSPIRE].

[48] T. Springer, Sound mode hydrodynamics from bulk scalar fields, Phys. Rev. D 79 (2009) 046003 [arXiv:0810.4354] [INSPIRE].

[49] T. Springer, Second order hydrodynamics for a special class of gravity duals, Phys. Rev. D 79 (2009) 086003 [arXiv:0902.2566] [INSPIRE].

[50] J.R. David, M. Mahato and S.R. Wadia, Hydrodynamics from the D1-brane, JHEP 04 (2009) 042 [arXiv:0901.2013] [INSPIRE].

[51] C. Wu, Y. Chen and M. Huang, Fluid/gravity correspondence: a nonconformal realization in compactified D4 branes, Phys. Rev. D 93 (2016) 066005 [arXiv: 1508.04038] [INSPIRE].

[52] G.D. Moore and K.A. Sohrabi, Thermodynamical second-order hydrodynamic coefficients, JHEP 11 (2012) 148 [arXiv:1210.3340] [INSPIRE].

[53] D. Mateos, R.C. Myers and R.M. Thomson, Thermodynamics of the brane, JHEP 05 (2007) 067 [hep-th/0701132] [INSPIRE].

[54] M. Haack and A. Yarom, Universality of second order transport coefficients from the gauge-string duality, Nucl. Phys. B 813 (2009) 140 [arXiv:0811.1794] [InSPIRE].

[55] P. Romatschke, Relativistic viscous fluid dynamics and non-equilibrium entropy, Class. Quant. Grav. 27 (2010) 025006 [arXiv:0906.4787] [INSPIRE].

[56] N. Banerjee, J. Bhattacharya, S. Bhattacharyya, S. Jain, S. Minwalla and T. Sharma, Constraints on fluid dynamics from equilibrium partition functions, JHEP 09 (2012) 046 [arXiv: 1203.3544] [INSPIRE].

[57] S.S. Gubser, Symmetry constraints on generalizations of Bjorken flow, Phys. Rev. D 82 (2010) 085027 [arXiv: 1006.0006] [INSPIRE].

[58] S.S. Gubser and A. Yarom, Conformal hydrodynamics in Minkowski and de Sitter spacetimes, Nucl. Phys. B 846 (2011) 469 [arXiv:1012.1314] [InSPIRE].

[59] H. Marrochio, J. Noronha, G.S. Denicol, M. Luzum, S. Jeon and C. Gale, Solutions of conformal Israel-Stewart relativistic viscous fluid dynamics, Phys. Rev. C 91 (2015) 014903 [arXiv: 1307.6130] [INSPIRE].

[60] Y. Hatta, J. Noronha and B.-W. Xiao, Exact analytical solutions of second-order conformal hydrodynamics, Phys. Rev. D 89 (2014) 051702 [arXiv:1401.6248] [INSPIRE].

[61] Y. Hatta, J. Noronha and B.-W. Xiao, A systematic study of exact solutions in second-order conformal hydrodynamics, Phys. Rev. D 89 (2014) 114011 [arXiv:1403.7693] [INSPIRE].

[62] M.I. Nagy, T. Csorgo and M. Csanad, Detailed description of accelerating, simple solutions of relativistic perfect fluid hydrodynamics, Phys. Rev. C 77 (2008) 024908 [arXiv:0709.3677] [INSPIRE].

[63] J. Ze-Fang, Y. Chun-Bin, M. Csanad and T. Csorgo, Accelerating hydrodynamic description of pseudorapidity density and the initial energy density in $p+p, C u+C u, A u+A u$ and $P b$ $+\mathrm{Pb}$ collisions at energies available at the BNL Relativistic Heavy Ion Collider and the CERN Large Hadron Collider, Phys. Rev. C 97 (2018) 064906 [arXiv:1711.10740] [INSPIRE]. 